\title{
Multiresolution analysis as a criterion for effective dynamic mesh adaptation - A case study for Euler equations in the SAMR framework AMROC
}

\author{
Ralf Deiterding, ${ }^{\mathrm{a}, *}$ Margarete O. Domingues ${ }^{\mathrm{b}}$, Kai Schneider ${ }^{\mathrm{c}}$ \\ ${ }^{a}$ Aerodynamics and Flight Mechanics Research Group, University of Southampton, \\ Boldrewood Innovation Campus, SO16 7QF, United Kingdom \\ ${ }^{b}$ Associate Laboratory of Computing and Applied Mathematics, National Institute of \\ Space Research (INPE), Av. dos Astronautas, 1758, São José dos Campos, São Paulo, \\ 12.227-010, Brazil \\ ${ }^{c}$ Institut de Mathématiques de Marseille (I2M), Aix-Marseille Université, CNRS, 39 rue \\ Joliot-Curie, 13453 Marseille cedex 13, France
}

\section{Abstract}

Dynamic mesh adaptation methods require suitable refinement indicators. In the absence of a comprehensive error estimation theory, adaptive mesh refinement (AMR) for nonlinear hyperbolic conservation laws, e.g. compressible Euler equations, in practice utilizes mainly heuristic smoothness indicators like combinations of scaled gradient criteria. As an alternative, we describe in detail an easy to implement and computationally inexpensive criterion built on a two-level wavelet transform that applies projection and prediction operators from multiresolution analysis. The core idea is the use of the amplitude of the wavelet coefficients as smoothness indicator, as it can

\footnotetext{
*Corresponding author: Tel.: +44-23-8059-3384

Email addresses: r.deiterding@soton.ac.uk (Ralf Deiterding), margarete.domingues@inpe.br (Margarete O. Domingues), kai.schneider@univ-amu.fr (Kai Schneider)
} 
be related to the local regularity of the solution. Implemented within the fully parallelized and structured adaptive mesh refinement (SAMR) software system AMROC (Adaptive Mesh Refinement in Object-oriented C++), the proposed criterion is tested and comprehensively compared to results obtained by applying the scaled gradient approach. A rigorous quantification technique in terms of numerical adaptation error versus used finite volume cells is developed and applied to study typical two- and three-dimensional problems from compressible gas dynamics. It is found that the proposed multiresolution approach is considerably more efficient and also identifies besides discontinuous shock and contact waves - in particular smooth rarefaction waves and their interaction as well as small-scale disturbances much more reliably. Aside from pathological cases consisting solely of planar shock waves, the majority of realistic cases show reductions in the number of used finite volume cells between 20 to $40 \%$, while the numerical error remains basically unaltered.

Keywords:

Block-structured parallel adaptive mesh refinement, adaptation criteria, multiresolution analysis, wavelets, compressible Euler equations, AMROC

\section{Introduction}

In the numerical simulation of inviscid compressible gas dynamics, dynamic mesh adaptation based on flow features, especially shock waves and contact discontinuities, can reduce the computing time significantly, while preserving high accuracy of the numerical solutions. A recent benchmark of mesh adaptation techniques for the Euler equations on Cartesian meshes is 
given in [1]. A core finding of this work was that the wavelet-based multiresolution mesh adaptation approach, which is mathematically rigorous, leads to a more reliable mesh adaptation; yet, the block-based adaptation approach - thanks to very effective data structures - reduces the overall computing time drastically. Hence, we decided to combine both techniques into a single parallel computer code.

Multiresolution (MR) analysis, introduced by Mallat and Meyer [2, 3, 4], is intimately related to the theory of discrete wavelets and the development of the fast wavelet transform. Thus wavelet bases can be constructed which have some specific desired proprieties, like, for instance, (bi-)orthogonality, compact support, local regularity detection and norm equivalences. The idea of multiresolution analysis is to represent a function, or a flow field, at different resolution levels, which yield corresponding approximations with a finite number of mesh points. The approximation spaces are nested and can be generated by refinable functions. Wavelets then come into play when considering the difference between subsequent resolution levels. Computing the differences from the finest down to the coarsest resolution transforms a single-level representation of a function into a multiresolution representation. Mathematical tools from approximation theory allow to define adaptive (also called nonlinear) approximations of functions selecting only the most significant coefficients of their series representation. Such approximations are attractive as they reduce the number of coefficients and in addition provide rigorous estimations of the error to represent the function discretely, cf. [5]. Since the late 1980's such multiresolution representations have become very popular in a wide range of applications for data compression, de-noising and 
more recently also in adaptive computations of nonlinear partial differential equations (PDE). A key feature is suitable thresholding of the wavelet coefficients combined with an adaptation strategy to predict the set of active wavelet coefficients, which thus allows reducing the computational cost in terms of CPU time and memory requirements.

A detailed review of the literature using MR techniques for adaptive discretizations of PDEs is beyond the scope of the paper and we refer the interested reader for instance to $[6,7,8]$. A first application of MR techniques to PDEs was given by Harten who introduced MR analysis as an indicator to control the switch between cheap and expensive numerical fluxes on static fully refined grids [9]. Later on, these ideas were also applied to trigger local grid adaptation for hyperbolic conservation laws with the objective of automatic approximation error control and CPU time reductions. Harten's contributions were considered as seminal in this area too, with numerous theoretical and practical works carried out in order to verify the properties and efficiency of that approach, and also its extension to higher dimensions, cf. $[10,11,12]$. In the context of Uncertainty Quantification (UQ) for compressible flows MR techniques have been used in [13]. The main components of Harten's method is that the designed MR tool has two local operators to perform the wavelet transform, namely prediction and projection. These local operators can be combined to generate the so-called wavelet coefficients, which encode the information needed to go from a coarse to a finer resolution. The amplitudes of the wavelet coefficients can be used as local regularity indicators of the numerical solution. In regions where their amplitudes are small, the solution is smooth, while in regions where the amplitudes are large, the 
solution needs finer resolution. Hence, the wavelet coefficients are the basis of a natural refinement indicator and could also be used for mesh coarsening, if required by the adaptation approach.

In the context of discontinuous Galerkin methods multiresolution techniques have also been developed for grid adaptation in numerous works, for instance $[14,15,16]$. For high-order DG discretizations multi-wavelets have been introduced for grid adaptation, which allow for higher-order vanishing moments, while maintaining local support. Applications of adaptive simulations have been presented by Gerhard et al. for compressible flows [17].

In the framework of semi-Lagrangian methods, multiresolution analysis has been developed for triggering adaptive meshes for vortex methods by Rossinelli et al. [18], including local time stepping. A multi-code implementation for computing incompressible viscous flows in two space dimensions has been proposed in the open source package MRAG-I2D [18]. More recently, Tanaka et al. [19] enhanced a moving particle semi-implicit method using multiresolution tools and presented verification tests for channel and free surface flows.

In our work [1], a serial two- and three-dimensional implementation of MR smoothness detection in the cell-based finite volume code Carmen was compared with the scaled gradient $(\mathrm{SG})$ refinement criterion, as implemented in the AMROC (Adaptive Mesh Refinement in Object-oriented $\mathrm{C}++$ ) framework [20]. Note that AMROC uses a block-structured adaptive mesh refinement approach, while Carmen refines individual cells. In addition, the employed numerical fluxes and time integration strategies - while similar were not exactly identical. Hence, a direct comparison of refinement criteria 
in terms of numerical error and run-time performance was complicated as not only slightly different finite volume base schemes but also two different refinement algorithms on vastly different data structures were involved. Also, AMROC is fully parallelized, while the cell-based Carmen code was available in a serial version only, thereby restricting the comparison to smaller configurations. Still, the main results indicated that the MR approach presents a better localization of the adaptive solution, while the AMROC framework was roughly ten times faster than the Carmen code.

This motivated incorporation of the MR strategy as a refinement approach into AMROC [21]. The aim of the current work is to use one framework only, and to this end the multiresolution approach has been implemented into AMROC as a criterion for mesh refinement. Moreover, the fully parallel version of AMROC has been used together with block-based grids with hierarchical time step reduction. The underlying finite volume discretization of the SAMR and MR computations is now always identical and allows to assess the relative performance of scaled gradients and multiresolution based mesh refinement. Then, here, we use the new software system to report about the first comprehensive assessment of the MR approach for various adaptive shock-capturing schemes for Euler equations of compressible gas dynamics that have been previously implemented in AMROC [20].

The paper is organized as follows: Section 2 sketches the principles of SAMR for the Euler equations, its implementation in the AMROC framework, and especially details the different refinement indicators that are evaluated in this work. Then, a large set of $2 \mathrm{~d}$ as well as $3 \mathrm{~d}$ numerical experiments are presented in Section 3; conclusions and perspectives of this 
study are drawn in Section 4. In Appendix A a detailed description of the multiresolution approach for finite volumes is given.

\section{Structured adaptive mesh refinement}

\subsection{Governing equations for compressible gas dynamics}

In the solution of nonlinear hyperbolic partial differential equations, $\partial_{t} \mathbf{q}+$ $\nabla \cdot \mathbf{f}(\mathbf{q})=\mathbf{0}$, a multitude of length scales is ubiquitous. It is well established that for nonlinear flux functions $\mathbf{f}(\mathbf{q})$ even continuous initial data can develop into discontinuities over a finite time interval [22]. Here, we consider the three-dimensional compressible Euler equations written in conservation form with flux function $\mathbf{f}=\left(f_{1}, f_{2}, f_{3}\right)^{T}$ given by

$$
f_{1}=\left[\begin{array}{c}
\rho v_{1} \\
\rho v_{1}^{2}+p \\
\rho v_{1} v_{2} \\
\rho v_{1} v_{3} \\
(\rho E+p) v_{1}
\end{array}\right], f_{2}=\left[\begin{array}{c}
\rho v_{2} \\
\rho v_{1} v_{2} \\
\rho v_{2}^{2}+p \\
\rho v_{2} v_{3} \\
(\rho E+p) v_{2}
\end{array}\right], f_{3}=\left[\begin{array}{c}
\rho v_{3} \\
\rho v_{1} v_{3} \\
\rho v_{2} v_{3} \\
\rho v_{3}^{2}+p \\
(\rho E+p) v_{3}
\end{array}\right]
$$

with $\rho$ denoting the fluid density, $v_{i}$ are the components of the velocity vector and $E$ is the energy per unit mass. The hydrodynamic pressure $p$ for a perfect gas is given by the equation of state $p=(\gamma-1) \rho\left(E-\frac{\mathbf{v}^{2}}{2}\right)$, with $\gamma$ denoting the specific heat ratio. In the case of Euler equations discontinuous shock and contact waves can develop. Finite volume shock-capturing methods have been constructed to handle particularly this behavior in a robust and oscillation-free way [23]. Since in practical inviscid problems such discontinuities are usually very localized, a local increase of mesh resolution is beneficial to represent these jumps as accurately as possible. 


\subsection{Block-structured adaptive mesh refinement in $A M R O C$}

A particular important mesh adaptation approach for finite volume methods of hyperbolic conservation laws is the SAMR method after Berger and Collela $[24,25,26]$. This approach follows a patch-oriented strategy, where non-overlapping rectangular sub-meshes $G_{\ell, m}$ are employed to cover the do-

main $G_{\ell}$ of each level with index $\ell=0, \ldots, L$ as $G_{\ell}:=\bigcup_{m=1}^{M_{\ell}} G_{\ell, m}$. As the construction of refinement proceeds recursively, a hierarchy of sub-meshes, successively contained within the next coarser level domain, is created, cf. Fig. 1. Values of cells covered by finer sub-meshes are subsequently overwritten by averaged fine mesh values, which, in general, would lead to a loss of conservation on the coarser mesh. A remedy to this problem is to replace the coarse mesh numerical fluxes at refinement boundaries with the sum of fine mesh fluxes along the corresponding coarse cell boundary, cf. [25, 20].

The recursive nature of the algorithm allows only the addition of one new level in each refinement operation. The patch-based approach does not require special coarsening operations; sub-meshes are simply removed from the hierarchy. The coarsest possible resolution is thereby restricted to the level $\ell=0$ mesh. The resolution $\Delta x_{n, 0}$ and $\Delta t_{0}$ of the mesh at $\ell=0$ is specified by the user. In AMROC and most other SAMR implementations, all mesh widths on level $\ell$ are $r_{\ell}$-times finer than on the level $\ell-1$, i.e. $\Delta t_{\ell}=\Delta t_{\ell-1} / r_{\ell}$ and $\Delta x_{n, \ell}=\Delta x_{n, \ell-1} / r_{\ell}$, with $r_{\ell} \in \mathbb{N}, r_{\ell} \geq 2$ for $\ell>0$, which ensures that a time-explicit finite volume (FV) scheme remains stable under a CFL-type condition on all levels of the hierarchy.

Although AMROC allows arbitrary refinement factors, in order to stay within the framework of traditional multiresolution analysis, all computa- 
tions in this paper use dyadic grids with $r_{\ell}=2$ being used for the refinement of all levels.

The numerical update is applied on the level $\ell$ by calling a single-block routine implementing the FV scheme in a loop over all sub-meshes $G_{\ell, m}$. The regularity of the input data allows a straightforward implementation of the scheme and further permits optimization to take advantage of high-level caches, pipelining, etc. New refinement meshes are initialized by interpolating the vector of conservative quantities $\mathbf{Q}$ from the next coarser level; data in cells already refined are copied directly from the previous refinement patches. Ghost cells around each patch are used to decouple the sub-meshes computationally. The execution of the numerical loop in UpdateLevel() in Algorithm 1 requires the previous setting of the ghost cell values. Ghost cells outside of the root domain $G_{0}$ are used to implement physical boundary conditions. Ghost cells in $G_{\ell}$ have a unique interior cell analogue and are set by copying the data value from the patch where the interior cell is contained (synchronization). For $\ell>0$, internal boundaries can also be used. If recursive time step refinement is employed, ghost cells at the internal refinement boundaries on the level $\ell$ are set by time-space interpolation from the two previously calculated time steps of level $\ell-1$. Otherwise, spatial interpolation from the level $\ell-1$ is sufficient.

Besides data structures that store the topology of the hierarchy (cf. Fig. 1), the SAMR method requires at most two regular arrays assigned to each subgrid which contain the discrete vector of state $\mathbf{Q}$ for the actual and updated time step. In the Algorithms 1 and 2 we denote by $\mathbf{Q}^{\ell}(t)$ and $\mathbf{Q}^{\ell}\left(t+\Delta t_{\ell}\right)$ the unions of these arrays on level $\ell$. The edge- or face-centered 

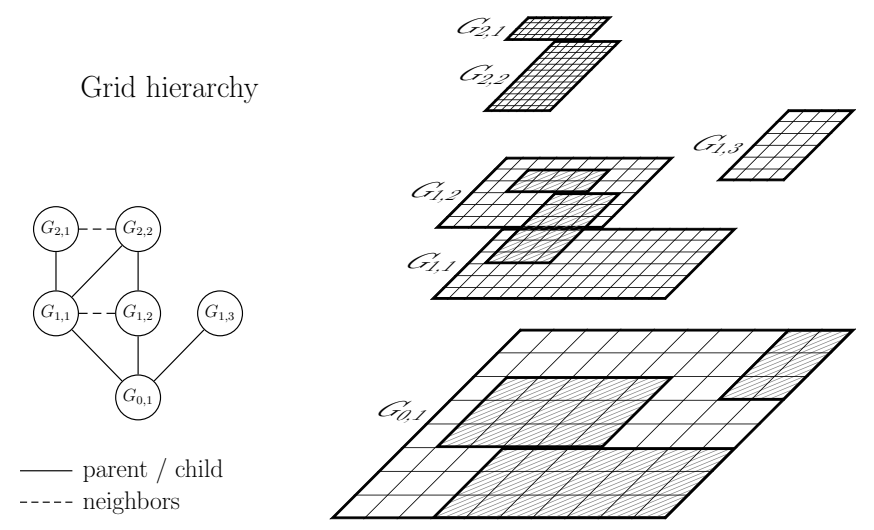

Figure 1: Hierarchy of rectangular sub-meshes in the SAMR approach.

flux correction terms $\delta \mathbf{F}^{n, \ell+1}$ have to be stored along the boundaries, where a level $\ell>0$ abuts the next coarser level. Initialization and calculation of the correction terms can be done efficiently during the loop over all subgrids in UpdateLevel(). The numerical fluxes $\mathbf{F}^{n}$ are necessary only temporarily.

New refinement grids on all higher levels are created when Regrid $(\ell)$ is called in Algorithm 1. Level $\ell$ itself is not modified. To consider the nesting of the level domains already in the grid generation, Algorithm 2 starts at the highest refinable level $\ell_{c}$, where $0 \leq \ell_{c}<L$. The refinement flags are stored in grid-based integer arrays $N^{\iota}$. Central to the block-structured mesh refinement approach is the utilization of a dedicated algorithm to create blocks from individual cells tagged for refinement by any of the criteria described in the next section. We use a recursive algorithm proposed by Bell et al. [27] to create a new block refinement $\breve{G}_{\iota+1}$ on basis of $N^{\iota}$. This method, inspired by techniques used in image detection, counts the number of flagged cells in each row and column on the entire domain. The sums $\Upsilon$ are called signatures. First, cuts into new boxes are placed on all edges where $\Upsilon$ is equal to zero. 
Algorithm 1: Recursive AMR algorithm.

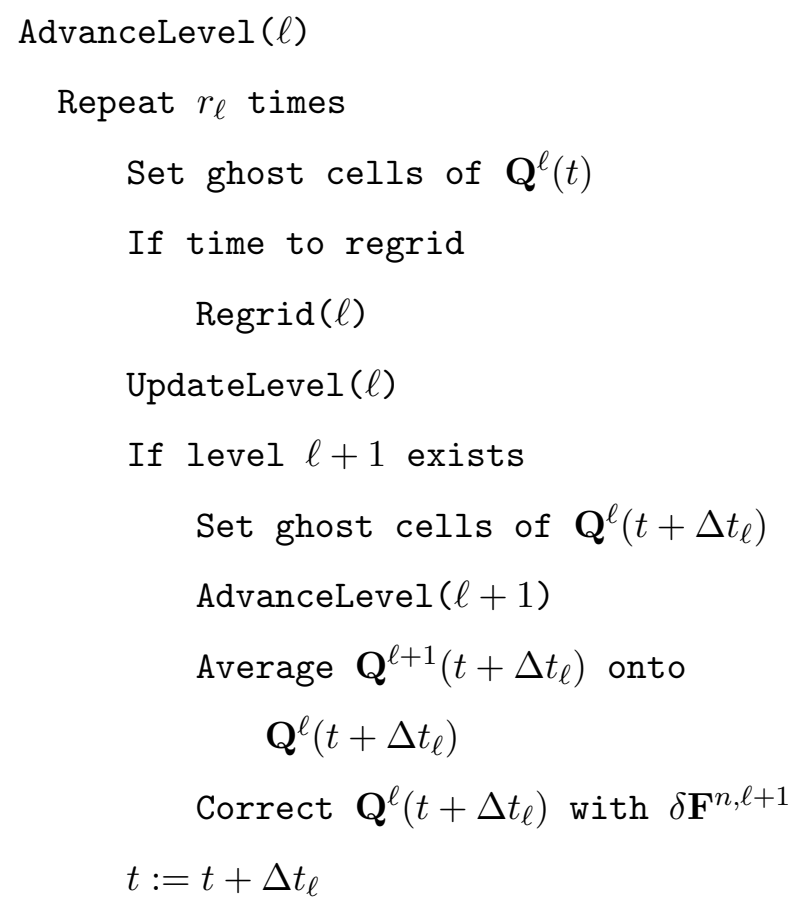

In the second step, cuts are placed at zero crossings of the discrete second derivative $\Delta=\Upsilon_{\nu+1}-2 \Upsilon_{\nu}+\Upsilon_{\nu-1}$. The algorithm starts with the steepest zero crossing and uses recursively weaker ones, until the ratio between flagged and all cells in every new mesh is above the prescribed threshold value $0<\eta \leq 1$. In practice, values around $\eta=0.80$ are used. A buffer zone of one or two cells is usually added around tagged cells to avoid degradation of results from interpolation. A depiction of the signatures, second derivatives and resulting blocks is given in Fig. 2. The upper index to $\Upsilon$ and $\Delta$ indicates the respective step of the recursive block generation procedure.

The re-initialization of the hierarchical data structures is done in the second loop of Algorithm 2 utilizing auxiliary data $\breve{\mathbf{Q}}^{\iota}(t)$. Cells in newly refined 


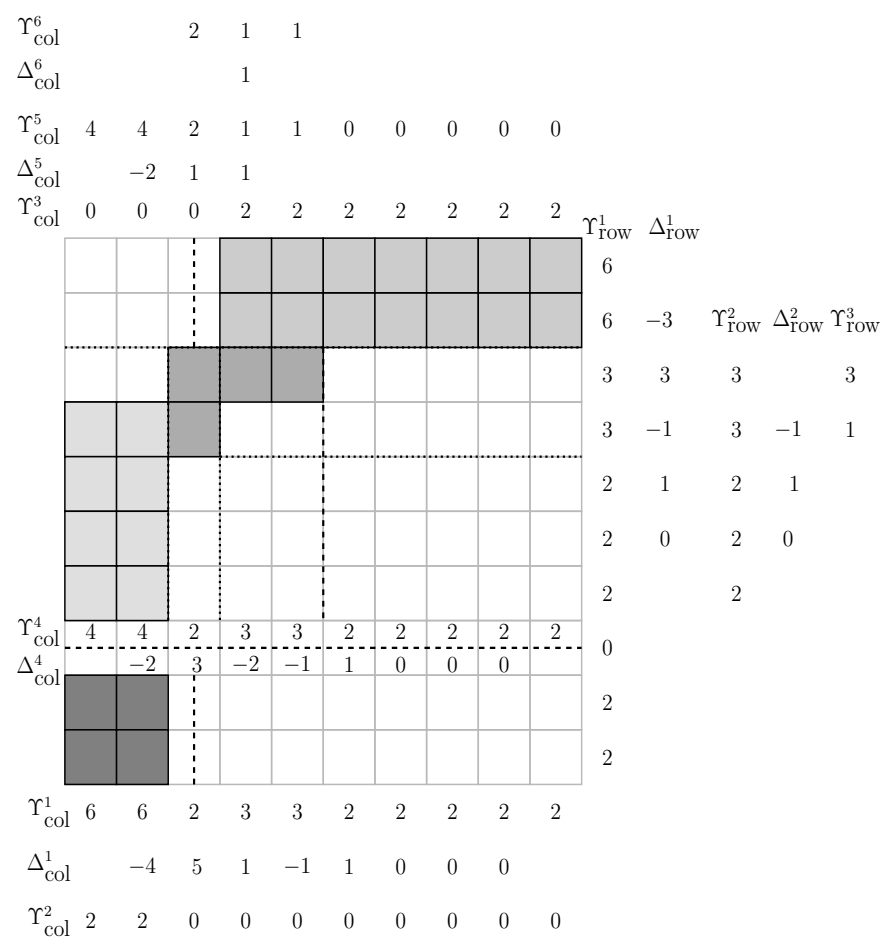

Figure 2: Signatures and second derivatives used for clustering.

regions $\breve{G}_{\iota} \backslash G_{\iota}$ are initialized by interpolation, values of cells in $\breve{G}_{\iota} \cap G_{\iota}$ are copied. As interpolation requires the previous synchronized reorganization of $\mathbf{Q}^{\iota-1}(t)$, recomposition starts on level $\ell+1$.

Our AMROC framework $[28,20]$ implements the SAMR method discretization-independent in one to three space dimensions and is fully parallelized for distributed memory systems. A rigorous domain decomposition strategy is pursued, in which the workload from all refinement levels is projected onto the level-0 cells before partitioning. The updated roughly load balancing distribution is then computed for level 0 only and all higher level subgrids are possibly split, redistributed across the parallel machine, and merged 
Algorithm 2: Regridding procedure.

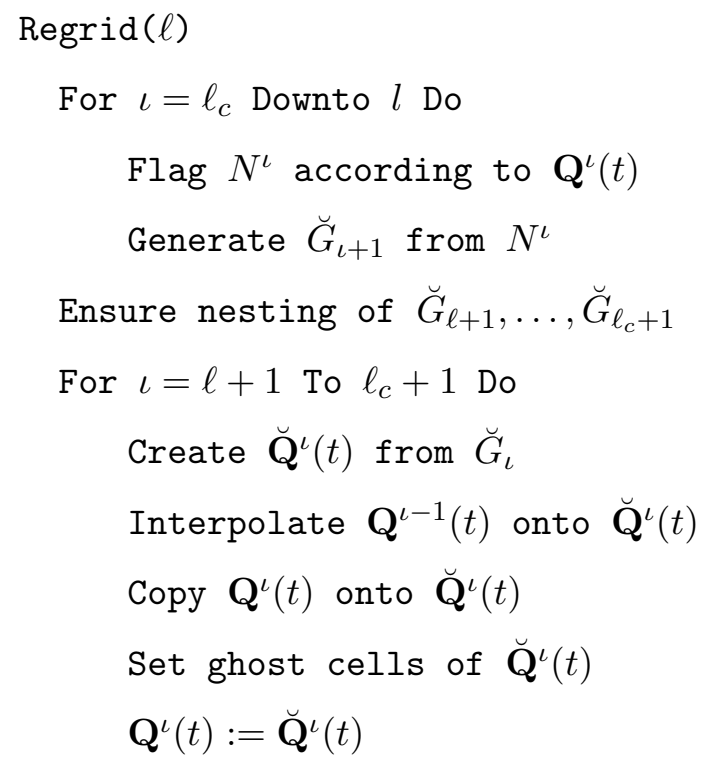

where possible, based on the new level-0 partition [29]. During repartitioning, all inter-processor communication patterns are updated. Overlapping ghost-cell regions of neighboring patch blocks are synchronized over processor borders as boundary conditions are applied using a single non-blocking MPI-library-call with all the ghost cell data between two respective processors. In AMROC, a generalized Hilbert space filling curve algorithm is used for load-balanced SAMR data distribution. On adaptive Cartesian meshes, space filling curves provide an effective compromise between the competing partitioning requirements of balancing the estimated workload and reducing the partition surface area for parallel ghost cell synchronization. Space filling curves are also locality preserving and hence induce only moderate data redistribution costs as the adaptive mesh is evolving. The present version of AMROC achieves close to linear scalability in dynamically adaptive $3 \mathrm{~d}$ 
simulations on $O(1000)$ processors.

\subsection{Standard refinement criteria}

In adaptive mesh refinement methods typically scaled gradient and heuristic error estimations are used. For example adaptation along discontinuities can be easily achieved by evaluating gradients multiplied by the step size in all spatial directions. For the sake of clarity we restrict the subsequent description to mainly two space dimensions and thereby flag a cell $(i, j)$ for refinement if at least one of the relations

$$
\begin{aligned}
\left|w\left(\mathbf{Q}_{i+1, j}\right)-w\left(\mathbf{Q}_{i, j}\right)\right|>\epsilon^{w},\left|w\left(\mathbf{Q}_{i, j+1}\right)-w\left(\mathbf{Q}_{i, j}\right)\right|>\epsilon^{w} \\
\left|w\left(\mathbf{Q}_{i+1, j+1}\right)-w\left(\mathbf{Q}_{i, j}\right)\right|>\epsilon^{w}
\end{aligned}
$$

is satisfied for a scalar quantity $w$, which is derived from the vector of state $\mathbf{Q}^{\ell}(t)$ on level $\ell$. The constant $\epsilon^{w}$ denotes the prescribed refinement threshold.

As an alternative to scaled gradients, AMROC also supports heuristic error estimation by Richardson extrapolation as suggested originally by Berger and Oliger [24]. In this technique, the current solution is integrated forward tentatively by one time step with $\Delta t_{\ell}$ and coarsened by a factor of 2 . Simultaneously, the solution from the previous time step is coarsened also by a factor of 2 and integrated one time step with $2 \Delta t_{\ell}$. After this, the local error estimation of the scalar quantity $w$ is computed, where for actual applications a normalized version of that error with a prescribed tolerance needs to be employed [28]. The error estimation technique is intended as a refinement indicator in regions where the solution is smooth and in practice it is generally combined with a scaled gradient criterion, e.g., to capture the shock waves [20]. 
While implementing Richardson extrapolation in an adaptive code is rather complex, already our early quantitative comparisons of criteria, cf. [21], uncovered significant deficiencies of the Richardson error estimation technique in detecting smooth structures in oblique directions to the Cartesian mesh. As a result, Richardson estimation was found to be a dramatically less efficient adaptation criterion, even for problems with smooth solution, than the scaled gradient approach. Hence it is not considered here any further.

\subsection{Multiresolution as a refinement criterion}

As explained above, the underlying idea of multiresolution techniques for mesh adaptation in numerical schemes is based on representing the numerical solution on different resolution levels. Compression of the number of mesh points, corresponding to coarsening the mesh locally, can be obtained by checking what happens between subsequent mesh resolutions $[6,7,8]$.

We consider a discrete solution of a FV discretization as initial cell average data $\mathbf{Q}^{\ell+1}$ at level $\ell+1$. The principle of the cell average MR methods is the transformation of these data $\mathbf{Q}^{\ell+1}$ into an equivalent multiscale representation. For instance, in one decomposition level we have

$$
\mathbf{Q}^{\ell+1} \underset{\text { prediction }}{\stackrel{\text { projection }}{\rightleftharpoons}} \mathbf{Q}_{M R}^{\ell+1}=\left\{\mathbf{Q}^{\ell}\right\} \cup\left\{\mathbf{d}^{\ell+1}\right\}
$$

where the set $\mathbf{d}^{\ell+1}$ contains the information between the two consecutive levels $\ell$ and $\ell+1$, and $\mathbf{Q}^{\ell}$ stores a smoothed version of the original numerical solution $\mathbf{Q}^{\ell+1}$. The data at the highest resolution level are transformed into a set of coarser scale approximations plus a series of prediction errors 
corresponding to wavelet coefficients $\mathbf{d}^{\ell}$. These coefficients describe the information difference between subsequent resolutions. In order to perform the MR method with finite volume data, operations for projection and prediction are required, where the cell values are local averages. These local averages correspond in the wavelet language to the scaling functions using the Haar basis (cf. Appendix A).

A coarser cell $\Omega_{i, j}^{\ell}$ has its value estimated using the finer level values and a unique projection operator $P^{\ell+1 \rightarrow \ell}: \mathbf{Q}^{\ell+1} \mapsto \mathbf{Q}^{\ell}$. For the two-dimensional case [30], the projection is performed by

$$
\mathbf{Q}_{i, j}^{\ell}=\frac{1}{4}\left(\mathbf{Q}_{2 i, 2 j}^{\ell+1}+\mathbf{Q}_{2 i, 2 j+1}^{\ell+1}+\mathbf{Q}_{2 i+1,2 j}^{\ell+1}+\mathbf{Q}_{2 i+1,2 j+1}^{\ell+1}\right)
$$

where $\mathbf{Q}_{i, j}^{\ell}$ is the average value of the cell $\Omega_{i, j}^{\ell}$. Conversely, the prediction operators $P_{i, j}^{\ell \rightarrow \ell+1}: \mathbf{Q}^{\ell} \mapsto \mathbf{Q}^{\ell+1}$ yield a non-unique approximation of $\mathbf{Q}_{i, j}^{\ell+1}$ by interpolation. Following the line of argumentation of Appendix A, the two-dimensional operators using tensor products for $m, n \in\{0,1\}$ read

$$
\begin{aligned}
& \widehat{\mathbf{Q}}_{2 i+m, 2 j+n}^{\ell+1}=\mathbf{Q}_{i, j}^{\ell} \\
& \quad+\frac{1}{8}\left[(-1)^{m}\left(\mathbf{Q}_{i+1, j}^{\ell}-\mathbf{Q}_{i-1, j}^{\ell}\right)+(-1)^{n}\left(\mathbf{Q}_{i, j+1}^{\ell}-\mathbf{Q}_{i, j-1}^{\ell}\right)\right] \\
& \quad+\frac{1}{64}\left[(-1)^{m n}\left(\mathbf{Q}_{i+1, j+1}^{\ell}-\mathbf{Q}_{i+1, j-1}^{\ell}-\mathbf{Q}_{i-1, j+1}^{\ell}+\mathbf{Q}_{i-1, j-1}^{\ell}\right)\right]
\end{aligned}
$$

where the cell $\widehat{\mathbf{Q}}_{i, j}^{\ell+1}$ is an approximation of the value $\mathbf{Q}_{i, j}^{\ell}$ and its eight nearest cells $\mathbf{Q}_{i \pm 1, j \pm 1}^{\ell}$. With this choice, the operators satisfy the properties of locality and consistency.

Following Eq. (A.9), the prediction operator is used to obtain the wavelet coefficients $\mathbf{d}_{2 i, 2 j+1}^{\ell+1}, \mathbf{d}_{2 i+1,2 j}^{\ell+1}$ and $\mathbf{d}_{2 i+1,2 j+1}^{\ell+1}$, of the finer cells. The coefficients 
are given by the approximation error of the prediction operator as

$$
\begin{aligned}
\mathbf{d}_{2 i, 2 j+1}^{\ell+1} & =\mathbf{Q}_{2 i, 2 j+1}^{\ell+1}-\widehat{\mathbf{Q}}_{2 i, 2 j+1}^{\ell+1}, \mathbf{d}_{2 i+1,2 j}^{\ell+1}=\mathbf{Q}_{2 i+1,2 j}^{\ell+1}-\widehat{\mathbf{Q}}_{2 i+1,2 j}^{\ell+1}, \\
\mathbf{d}_{2 i+1,2 j+1}^{\ell+1} & =\mathbf{Q}_{2 i+1,2 j+1}^{\ell+1}-\widehat{\mathbf{Q}}_{2 i+1,2 j+1}^{\ell+1} .
\end{aligned}
$$

From wavelet theory it is known that the decay of the wavelet coefficients also estimates the local regularity of a function, and in our case, of the computed numerical solution [10]. Hence, it is natural to utilize the wavelet coefficients to determine dynamic refinement as they indicate regions of steep gradients or discontinuities. Since the objective of a refinement indicator is the creation of level $\ell+1$ from level $\ell$, the criterion needs to be evaluated on $\ell$ and we thereby have to shift Eq. (4) one level downward. Consequently, $\widehat{\mathbf{Q}}_{i, j}^{\ell}$ is obtained as the averaged projection onto the next coarser level $\ell-1$, which is interpolated again back onto level $\ell$, i.e., we evaluate the wavelet coefficients $\mathbf{d}_{i, j}^{\ell}=\mathbf{Q}_{i, j}^{\ell}-\widehat{\mathbf{Q}}_{i, j}^{\ell}$ in fact as

$$
\mathbf{d}_{i, j}^{\ell}=\mathbf{Q}_{i, j}^{\ell}-P_{0 / 1}^{\ell-1 \rightarrow \ell} P^{\ell \rightarrow \ell-1}\left(\mathbf{Q}_{i, j}^{\ell}\right)
$$

Comparing a suitable norm of the local wavelet coefficient with a userspecified regularity threshold, $\left|\mathbf{d}_{i, j}^{\ell}\right|>\epsilon$, then marks cells at level $\ell$ for refinement at the next finer level.

Based on the considered norm for the error estimate and depending on the space dimensions, there are different possible choices for the threshold, which allow identifying the retained large wavelet coefficients. In the context of finite volumes, in order to control the $L_{1}$-norm, Harten's thresholding strategy is primarily used, i.e,

$$
\epsilon^{\ell}=\frac{\epsilon}{|\Omega|} 2^{d(\ell+1-L)}, \quad 0 \leq \ell<L
$$


where $L$ is the finest scale level, the dimension parameter $d=2$ or 3 according to the dimension used, and $|\Omega|$ is the cell area. It is also possible to use a constant threshold value $\epsilon$ for all levels. For instance, in [31] it was found that for multiresolution techniques applied for UQ using a truncation and encode approach thresholding with constant values could be more efficient than the level dependent choice, proposed by Harten [32]. In the context of adaptive stochastic problems Abgrall et al. [33] used however a level dependent threshold. In Domingues et al. [34] we analyzed the choice of the threshold for the compressible Euler equations and found that level (and dimension) dependent values yield better results considering the MR category that combines a time adaptive strategy with a controlled time-stepping.

In some of the numerical experiments we use different threshold values to verify the influence of the perturbation error. In practical applications the threshold should be chosen such that perturbation (thresholding) and discretization errors are of the same order. One possible option for determining the order of the threshold value is to perform a series of computations on a coarser mesh. For linear advection and advection-diffusion equations a theoretical error estimate has been provided in $[35,12]$, however a constant needs to be estimated which also requires test computations, as discussed in [12].

While it seems natural to use a vector norm, e.g., the Euclidean norm, to compute $\left|\mathbf{d}_{i, j}^{\ell}\right|$ for the entire state vector in each cell, this usually requires very careful rescaling to ensure an equal consideration of all vector components in the refinement indicator. In practice, the application of multiple scalar refinement indicators with individual threshold values is generally easier to 
use. Since all flow features inherent to the Euler equations can be well detected in the density field, we have opted in this investigation to apply the new multiresolution criterion as well as the scalar gradient criterion to $\rho$ only. Just the benchmark of Section 3.3 used scalar criteria for $\rho$ and $p$ in combination.

The extension of the described criterion to three space dimensions is done in a canonical manner. First, the projection operation $P^{\ell+1 \rightarrow \ell}$ is formulated as the algebraic average of eight fine grid cells. Secondly, the interpolation operations $P_{i, j, k}^{\ell \rightarrow \ell+1}: \mathbf{Q}^{\ell} \mapsto \mathrm{Q}^{\ell+1}$ are constructed as tensor products. For the computations of Section 3.3 we have used the polynomial prediction operators given in [12], which reads for $m, n, p \in\{0,1\}$

$$
\begin{gathered}
\widehat{\mathbf{Q}}_{2 i+m, 2 j+n, 2 k+p}^{\ell+1}=\mathbf{Q}_{i, j, k}^{\ell}+\frac{1}{8}\left[(-1)^{m}\left(\mathbf{Q}_{i+1, j, k}^{\ell}-\mathbf{Q}_{i-1, j, k}^{\ell}\right)\right. \\
\left.+(-1)^{n}\left(\mathbf{Q}_{i, j+1, k}^{\ell}-\mathbf{Q}_{i, j-1, k}^{\ell}\right)+(-1)^{p}\left(\mathbf{Q}_{i, j, k+1}^{\ell}-\mathbf{Q}_{i, j, k-1}^{\ell}\right)\right] \\
+\frac{1}{64}\left[(-1)^{m n}\left(\mathbf{Q}_{i+1, j+1, k}^{\ell}-\mathbf{Q}_{i+1, j-1, k}^{\ell}-\mathbf{Q}_{i-1, j+1, k}^{\ell}+\mathbf{Q}_{i-1, j-1, k}^{\ell}\right)\right. \\
(-1)^{n p}\left(\mathbf{Q}_{i, j+1, k+1}^{\ell}-\mathbf{Q}_{i, j-1, k+1}^{\ell}-\mathbf{Q}_{i, j+1, k-1}^{\ell}+\mathbf{Q}_{i, j-1, k-1}^{\ell}\right) \\
\left.(-1)^{m p}\left(\mathbf{Q}_{i+1, j, k+1}^{\ell}-\mathbf{Q}_{i-1, j, k+1}^{\ell}-\mathbf{Q}_{i+1, j, k-1}^{\ell}+\mathbf{Q}_{i-1, j, k-1}^{\ell}\right)\right] \\
+\frac{1}{512}\left[\mathbf{Q}_{i+1, j+1, k+1}^{\ell}-\mathbf{Q}_{i+1, j+1, k-1}^{\ell}-\mathbf{Q}_{i+1, j-1, k+1}^{\ell}-\mathbf{Q}_{i-1, j+1, k+1}^{\ell}+\right. \\
\left.\mathbf{Q}_{i+1, j-1, k-1}^{\ell}+\mathbf{Q}_{i-1, j+1, k-1}^{\ell}+\mathbf{Q}_{i-1, j-1, k+1}^{\ell}-\mathbf{Q}_{i-1, j-1, k-1}^{\ell}\right]
\end{gathered}
$$

Finally, the extension of Eq. (5) to three dimensions defines seven wavelet 
coefficients

$$
\begin{aligned}
\mathbf{d}_{2 i, 2 j+1,2 k}^{\ell+1} & =\mathbf{Q}_{2 i, 2 j+1,2 k}^{\ell+1}-\widehat{\mathbf{Q}}_{2 i, 2 j+1,2 k}^{\ell+1}, \\
\mathbf{d}_{2 i+1,2 j, 2 k}^{\ell+1}= & \mathbf{Q}_{2 i+1,2 j, 2 k}^{\ell+1}-\widehat{\mathbf{Q}}_{2 i+1,2 j, 2 k}^{\ell+1}, \\
\vdots & \\
\mathbf{d}_{2 i+1,2 j+1,2 k+1}^{\ell+1}= & \mathbf{Q}_{2 i+1,2 j+1,2 k+1}^{\ell+1}-\widehat{\mathbf{Q}}_{2 i+1,2 j+1,2 k+1}^{\ell+1} .
\end{aligned}
$$

In AMROC, we compute the dimension-dependent version of Eq. (5) on a per-block basis using temporary auxiliary data coarsened by a factor of two. It is apparent that all computations are strictly local and thereby no additional parallelization effort or penalty is involved.

\subsection{Evaluation of the adaptation error}

In order to compare different refinement criteria quantitatively, it is necessary to evaluate the numerical error of an adaptive computation with respect to the error of the same computation on a uniform mesh of the same maximal resolution. Here, we evaluate the numerical error primarily in the discrete $L_{1}$ norm, which is introduced for the error on level $\ell$ on a level-wise domain $G_{\ell}$ as

$$
L_{1}\left(\mathbf{Q}^{\ell}, G_{\ell}\right)=\sum_{i, j}\left|\mathbf{Q}_{i, j}^{\ell}-\mathbf{q}\left(x_{i}, y_{j}\right)\right| \Delta x_{\ell} \Delta y_{\ell},
$$

with $\mathbf{q}\left(x_{i}, y_{j}\right)$ and $i, j \in \mathbb{Z}$ denoting the exact solution evaluated point-wise in the respective cell centers of a two-dimensional Cartesian grid. If the entire problem domain - that is $G_{0}$ in the SAMR approach - is refined at the maximal level $L$, we obtain the conventional numerical error of the numerical method on a uniform mesh with resolutions $\Delta x_{L}$ and $\Delta y_{L}$,

$$
L_{1, u n i}\left(\mathbf{Q}^{L}, G_{0}\right)=\sum_{i, j}\left|\mathbf{Q}_{i, j}^{L}-\mathbf{q}\left(x_{i}, y_{j}\right)\right| \Delta x_{L} \Delta y_{L}
$$


which we denote just by $L_{1, \text { uni }}(\mathbf{Q})$. In the adaptive case, however, the error on $G_{0}$ is given as the sum of the $L_{1}$-errors computed on those parts of $G_{\ell}$ that have no higher refinement, that is,

$$
L_{1}(\mathbf{Q})=L_{1}\left(\mathbf{Q}^{L}, G_{L}\right)+\sum_{\ell=0}^{L-1} L_{1}\left(\mathbf{Q}^{\ell}, G_{\ell} \backslash G_{\ell+1}\right) .
$$

In addition, we introduce the level-wise adaptation error $L_{1, A M R}\left(\mathbf{Q}^{\ell}, G_{\ell}\right)$ that is defined as

$$
L_{1, A M R}\left(\mathbf{Q}^{\ell}, G_{\ell}\right)=\sum_{i, j}\left|\mathbf{Q}_{i, j}^{\ell}-\mathbf{Q}_{i, j}^{r}\right| \Delta x_{\ell} \Delta y_{\ell},
$$

where $\mathbf{Q}_{i, j}^{r}$ corresponds to a solution computed on a uniform mesh with step sizes corresponding to the finest adaptive resolution at level $L$ and averaged onto the actual level $\ell$, in case its resolution is coarser. Finally, the adaptation error on the entire domain, $L_{1, A M R}(\mathbf{Q})$, is defined from Eq. (12) in analogy to $L_{1}(\mathbf{Q})$ from Eq. (11).

In these notations, obviously the relation

$$
L_{1}(\mathbf{Q}) \leq L_{1, A M R}(\mathbf{Q})+L_{1, u n i}(\mathbf{Q})
$$

holds true, yielding

$$
L_{1}(\mathbf{Q})-L_{1, u n i}(\mathbf{Q}) \leq L_{1, A M R}(\mathbf{Q}) .
$$

Since $L_{1, \text { uni }}(\mathbf{Q})$ is the error of the finite volume scheme itself and independent of any mesh refinement procedure, i.e. a constant when varying the refinement criterion, monotone behavior in $L_{1, A M R}(\mathbf{Q})$ will equally be preserved in $L_{1}(\mathbf{Q})$.

In the following we will use primarily $L_{1, A M R}^{\rho}:=L_{1, A M R}(\rho)$ to measure the accuracy and suitability of dynamically generated refinement at a defined 
MR, constant threshold

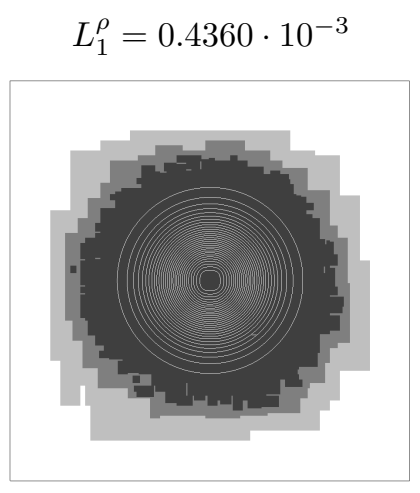

MR, hierarchical threshold

$$
L_{1}^{\rho}=0.4351 \cdot 10^{-3}
$$

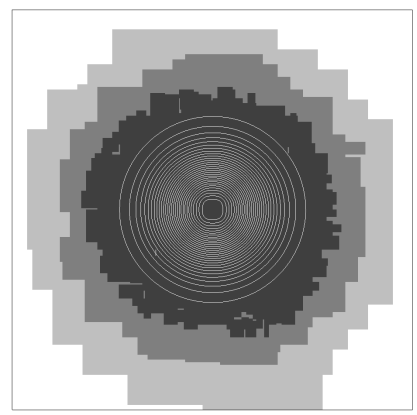

Scaled gradient

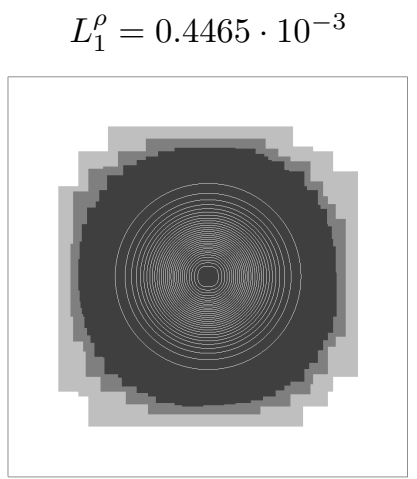

Figure 3: Advection of a Gaussian density bump refined with the different criteria after one period. Shown are isolines of density on regions of different refinement (depicted by different gray scales).

point in simulated time. The solution on the adapted mesh is compared to a reference solution computed at the maximal resolution following (12), where $\rho^{r}$ on coarser levels is generated from the density field of the uniform reference solution by simple averaging of higher level cell values, Eq. (2), as it is consistent with the finite volume approach. A post-processing tool has been written to carry out the required norm calculations using only AMROC output files.

\section{Computational experiments}

\subsection{Moving Gaussian bump}

In order to demonstrate the quantitative analysis approach, we first consider a simple two-dimensional test case with analytic solution. A Gaussian bump in density is advected with constant velocity and at constant pres- 


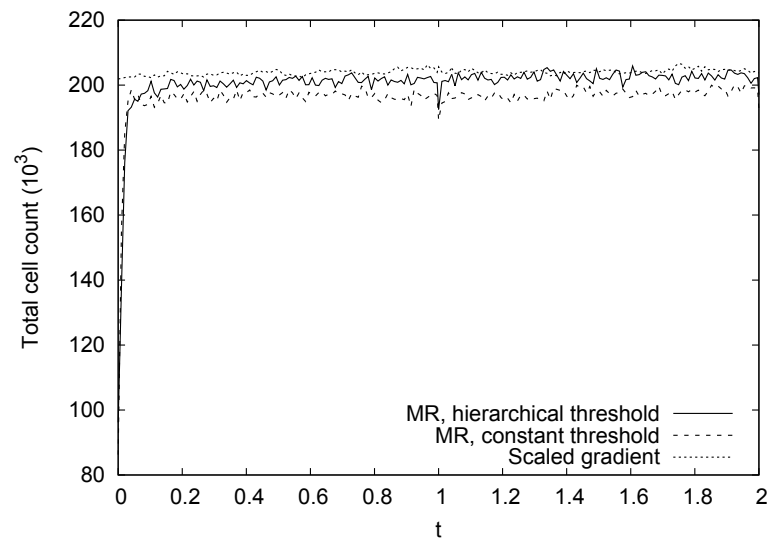

Figure 4: Total cell count over time for the cases from Fig. 3.

sure along the diagonal $x_{1}=x_{2}$. Periodic boundary conditions are used, which ensures that after exactly one propagation period, the exact solution does agree identically with the initial conditions, allowing easy evaluation of error norms. Further, this smooth test case will retain the maximal order of accuracy of any FV scheme, while the varying gradients of the solution lead to distinctively different adaptation patterns when the mesh refinement indicator is varied. The initial conditions read

$$
\rho\left(x_{1}, x_{2}\right)=1+\exp \left(-\frac{x_{1}^{2}+x_{2}^{2}}{R^{2}}\right)
$$

with $R=1 / 4$ and $v_{1}=v_{2} \equiv 1, p \equiv 1$. The domain size is $[-1,1] \times[-1,1]$ with periodic boundary conditions at all sides and the result is analyzed for a final time of $t_{\text {end }}=2$. A base mesh of $80 \times 80$ is used and three additional levels all refined by a factor 2 are applied. The used finite volume scheme is the Van Leer flux-vector splitting within a second-order-accurate MUSCL slopelimiting method combined with dimensional splitting. See [36] for details on these numerical techniques. The clustering efficiency is always $\eta=0.95$.

In Fig. 3, we present the final refinement regions (depicted by gray scales) 


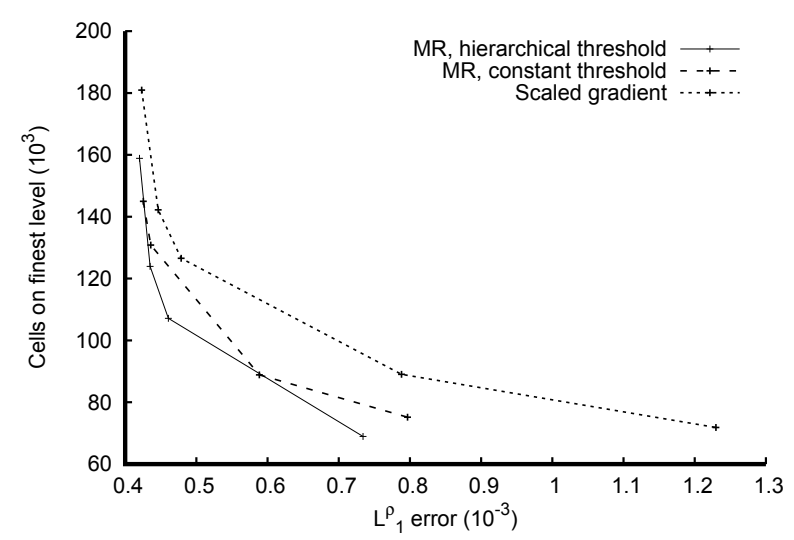

Figure 5: Cells on finest level versus $L_{1}^{\rho}$ error after one period.

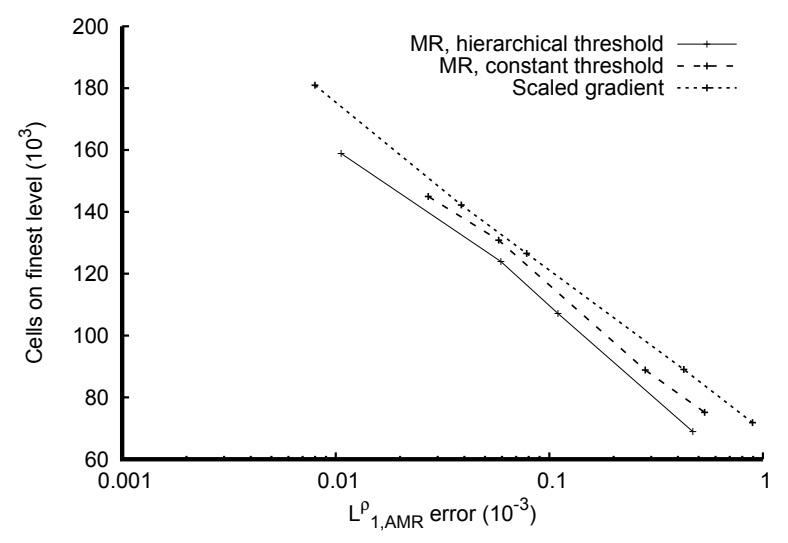

Figure 6: Cells on finest level vs. $L_{1, A M R}^{\rho}$ error after one period.

created by the scaled gradient criterion and the MR criterion applied in $\rho$ only with and without hierarchical threshold for an error $L_{1}^{\rho} \approx 0.44 \cdot 10^{-3}$ and $3 \cdot 10^{-5}<L_{1, A M R}^{\rho}<6 \cdot 10^{-5}$ in all three cases. Figure 4 visualizes the total cell count of the computations from Fig. 3 over time. Despite yielding a slightly larger error, the SG computation uses a larger cell number of cells effectively throughout the entire computation. For all three cases the number of cells in general increases throughout the computation, which is due to the 
Gaussian bump spreading from the numerical diffusion of the scheme and the mesh adapting accordingly. The MR criteria exhibit marginally larger variations in cell count than the SG calculation; however, all criteria provide reliable and stable mesh adaptation. It is interesting to note that the MR criteria create a considerable smaller higher level refinement based on the initial conditions. For these criteria, the cell count at level 2 plateaus after one to two level-0 time steps and remains roughly constant on level 3 only after the fourth level-0 time step.

Figures 5 and 6 plot the $L_{1}^{\rho}$ and $L_{1, A M R}^{\rho}$ errors respectively, for the refinement criteria applied to the density as the threshold value is varied. Displayed are the number of the cells on the finest level versus the error, as a measure for efficiency of the used refinement. The two figures show that the MR criteria are more efficient than the scaled gradient criterion, as the MR curves are always below the respective scaled gradient curve.

This monotonicity is clearly visible in $L_{1, A M R}^{\rho}$ and - following the argument from Section 2.5 - is even more apparent in $L_{1}^{\rho}$. Already this simple test demonstrates the excellent performance of the MR criteria. In subsequent tests, we will use primarily $L_{1, A M R}^{\rho}$ to quantify criteria performance. This is because the studied test cases do not have a known analytic solution and $L_{1}^{\rho}$ cannot easily be approximated. The test case of the moving Gaussian bump also illustrates that $L_{1, A M R}^{\rho}$ is a reliable and unambiguous measure for adaptation accuracy.

Each curve of Fig. 6 corresponds to a discrete mapping $\left\{\tau_{1}, \ldots, \tau_{M}\right\} \mapsto$ $\left\{N_{1}, \ldots, N_{M}\right\}$ involving $M$ computational experiments, where $\tau=\log _{10}\left(L_{1, A M R}^{\rho}\right)$ is introduced as the decadic logarithm of the measured numerical error value 
and $N$ is a suitable count of the cells used. In order to establish quantitative measures for comparing such maps, we denote as $g(\tau)$ the continuous function defined piecewise by

$$
g_{i}: \tau \mapsto N_{i}+\left(\tau-\tau_{i}\right) \frac{N_{i+1}-N_{i}}{\tau_{i+1}-\tau_{i}} \quad \text { for } \tau \in\left[\tau_{i}, \tau_{i+1}[\right.
$$

for all $i \in\{1, \ldots, M-1\}$. We compare the respective functions, e.g., for SG and MR criterion, $g_{S G}(\tau)$ and $g_{M R}(\tau)$, only within that interval $\left[\tau_{s}, \tau_{e}\right]$, in which both functions are defined, i.e. $\tau_{s}=\max \left\{\tau_{1}^{S G}, \tau_{1}^{M R}\right\}$ and $\tau_{e}=$ $\min \left\{\tau_{M}^{S G}, \tau_{M}^{M R}\right\}$. Using these definitions, we define a measure for the average cell count saving of the MR criteria versus SG as

$$
\Delta \bar{N}=\frac{1}{\tau_{e}-\tau_{s}} \int_{\tau_{s}}^{\tau_{e}}\left[g_{S G}(\zeta)-g_{M R}(\zeta)\right] d \zeta .
$$

The average efficiency of the MR criteria relative to the SG criterion is defined as

$$
\bar{E}=\frac{1}{\tau_{e}-\tau_{s}} \int_{\tau_{s}}^{\tau_{e}} \frac{g_{S G}(\zeta)-g_{M R}(\zeta)}{g_{S G}(\zeta)} d \zeta .
$$

A straightforward Python script has been written to compute Eqs. (17) and (18) from tabulated data. Using this tool, we obtain for the data of Fig. 6 $\Delta \bar{N}=6088$ and $\bar{E}=5.7 \%$ when comparing the MR criterion with constant threshold value to the SG criterion, and $\Delta \bar{N}=12645$ and $\bar{E}=10.4 \%$ for MR with hierarchical thresholding. The latter result means that for this problem the MR criterion with hierarchical thresholding will obtain a similar numerical accuracy as the SG criterion on finest meshes that are about 10\% smaller in cell count. 


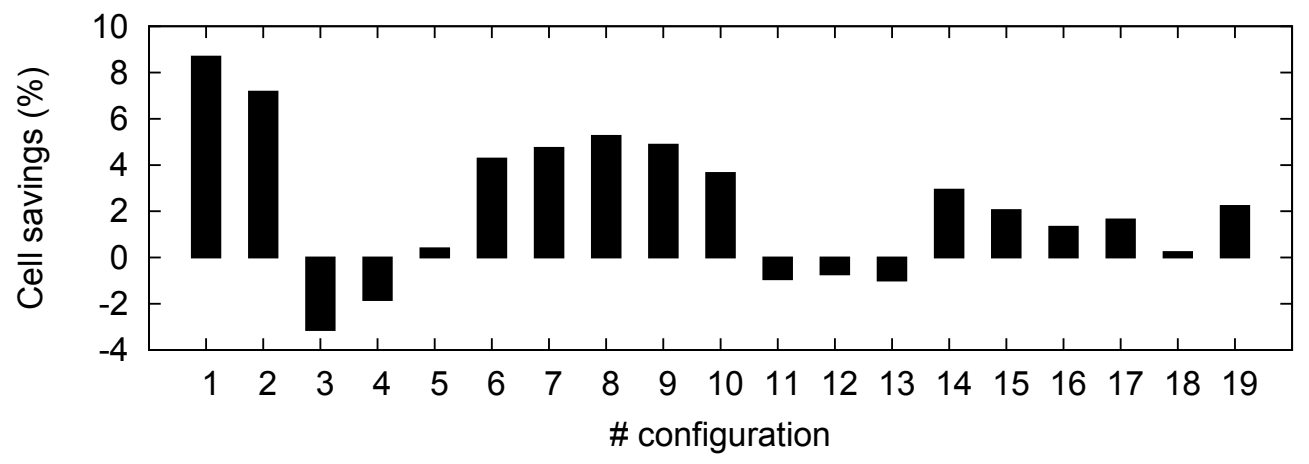

Figure 7: Average cells savings of MR versus SG with respect to the uniform case $\Delta \bar{N} / N_{\text {uni }}$ for the 19 Lax-Liu configurations; MUSCL scheme used.

\subsection{Two-dimensional Lax-Liu Riemann problems}

As comprehensive test cases for realistic gas dynamics, we employ the above defined measures to compare the MR criterion with hierarchical thresholding to the SG approach for the 19 classical two-dimensional Riemann problems for Euler equations described by Lax \& Liu in [37], as described in Appendix B. Depending on the initial values, planar waves can develop into complex $2 \mathrm{~d}$ patterns that give a good representation for a variety of flow phenomena intrinsic to the multi-dimensional Euler equations. For instance, the computational domain and the initial values for the configurations are presented in Fig. B.21 and Table B.4, respectively. The final simulated times are the same as reported by Lax \& Liu [37], cf. Table B.3.

In all computations the CFL number is kept roughly constant at 0.5 and the adiabatic exponent is set to the value $\gamma=1.4$. The main investigations use a standard unsplit shock-capturing MUSCL scheme with Minmod-limiter and Roe numerical flux function [38, 36], which employs the MUSCL-Hancock approach for time integration, yielding an overall second- 


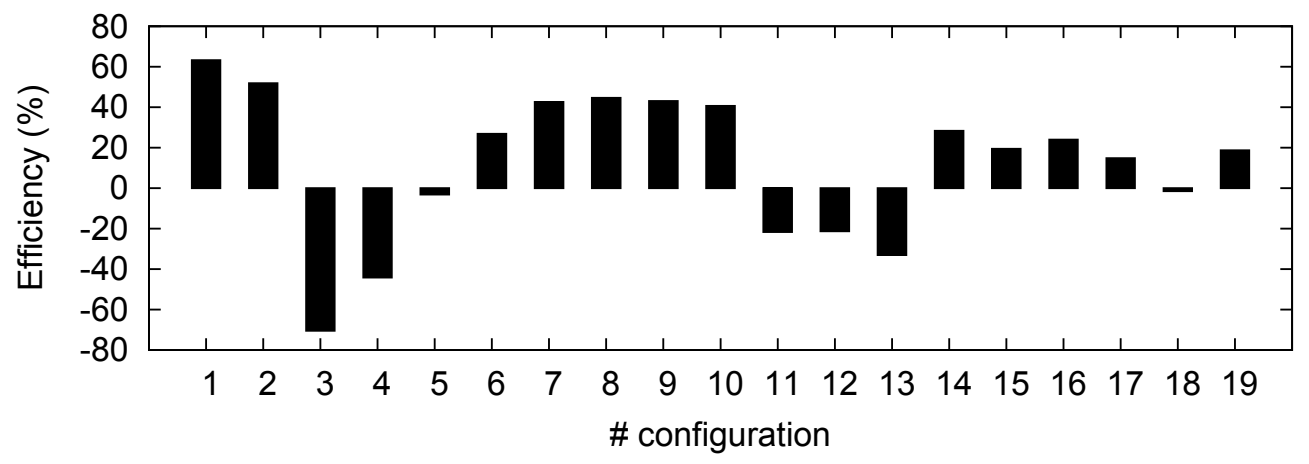

Figure 8: Average efficiency $\bar{E}$ of $M R$ with respect to the SG criterion for the 19 Lax-Liu configurations; MUSCL scheme used.

order-accurate numerical method [36]. For comparison, some calculations are in addition repeated with a WENO scheme with Roe flux function, which is third-order-accurate in space. This method uses a storage efficient explicit 3-stage Runge-Kutta scheme for time integration, yielding an overall third-order-accurate numerical scheme [39].

The base resolution of all computations is $64 \times 64$ cells and four additional levels, each refined by a factor 2 , are used. The adaptation criteria are again applied just in density $\rho$. For computing the adaptation error $L_{1, A M R}^{\rho}$ we consider a reference solution on the uniform mesh $1024 \times 1024$. We have tested the cluster parameters $\eta=0.75,0.80$, and 0.99 , and we also computed a distribution of $M=12$ threshold parameters for each adaptation criterion. For all configurations, we use MR with hierarchical threshold considering $L=5$. Since the compression results obtained with these cluster parameters show very similar trends, we present here just the results for $\eta=0.80$.

In general, both criteria identify the essential flow features and lead to sensibly adapted meshes; however, notable differences between the 19 differ- 
(a) Lax-Liu config \#3

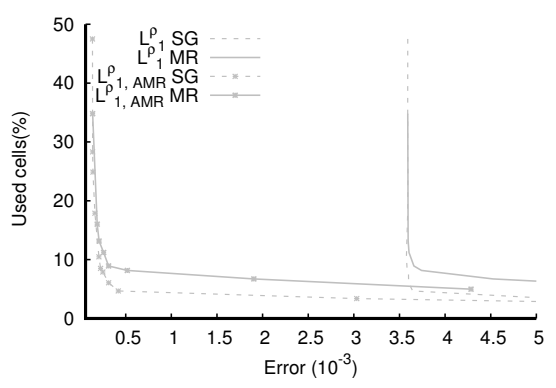

(b) Lax-Liu config \#6

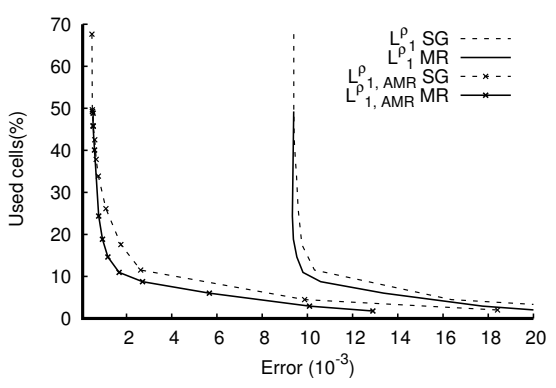

(c) Lax-Liu config \#10

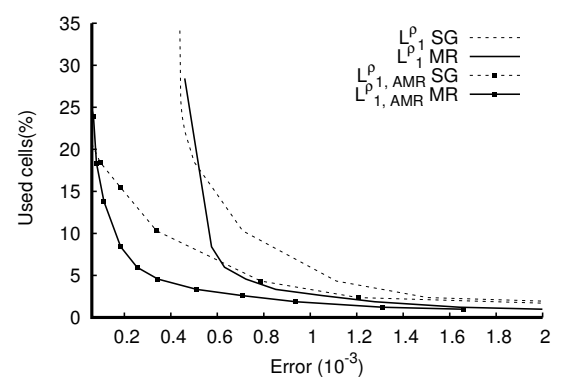

Figure 9: Used cells in the adaptive MUSCL computations vs. $L_{1}^{\rho}$ and $L_{1, A M R}^{\rho}$ error.

ences configurations can be observed. Using the number of cells on all SAMR levels and throughout all time steps as cell metric $N$, we have evaluated the average cell count saving, Eq. (17), and the average efficiency, Eq. (18), of MR versus SG for all 19 cases. Fig. 7 shows in percent the ratio $\Delta \bar{N} / N_{u n i}$ for the MUSCL scheme; Fig. 8 displays the average efficiency $\bar{E}$ of the MR criterion compared to the SG criterion directly. From Fig. 7 one deduces that in absolute values, MR presents clear benefits for 12 configurations (where six of them achieve $>4 \%$ of savings compared to the uniform case), is comparable 
(a) Lax-Liu config \#3

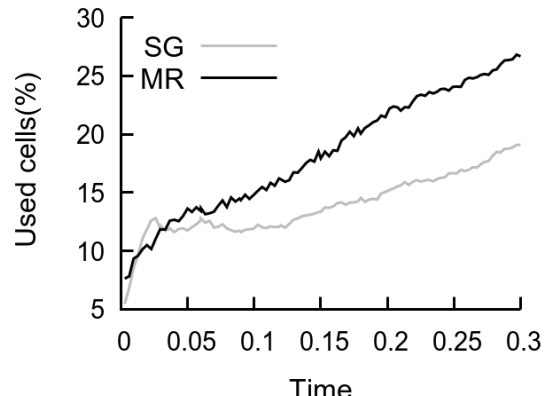

(b) Lax-Liu config \#6

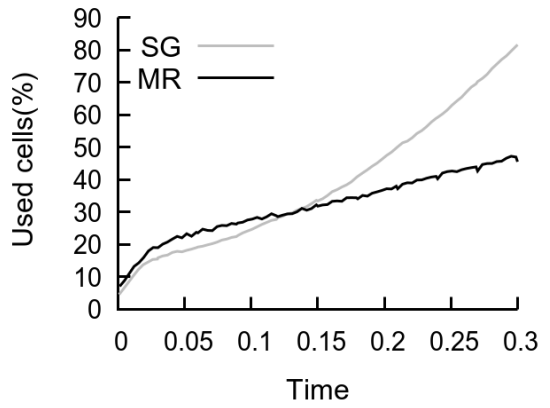

(c) Lax-Liu config \#10

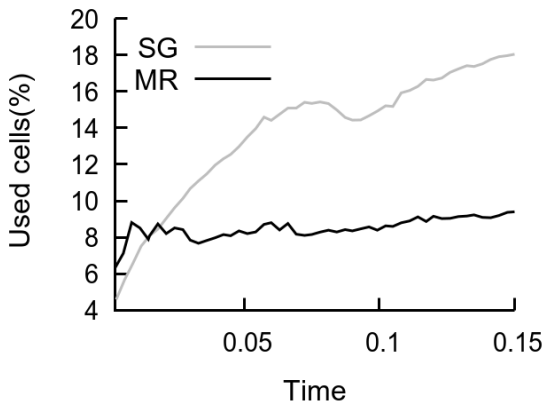

Figure 10: Time evolution of the used cells for the adaptive MUSCL computations listed in Table 1.

to SG in five cases, and performs notably worse for just two configurations. In relative terms, Fig. 8 shows 11 average efficiencies around and larger than $20 \%$ in favour of the MR criterion and five cases with values smaller than $-20 \%$, for which the SG approach performs more effectively.

A closer analysis of the occurring flow patterns uncovers that the MR criterion obtains its highest efficiencies for cases dominated by widespread rarefaction waves (Configs. \#1 and \#2). In these configurations more than $7 \%$ of the cells on the whole uniform mesh are saved. In Configs. \#6-10 there are rarefaction waves interacting with shear layers, i.e. contact discontinuities with different transverse velocities. For these cases the MR criteria also 
perform consistently and considerably better than SG, capturing all relevant flow structures reliably and efficiently.

In situations in which shock waves in addition interact with rarefaction waves and/or contact lines (like Configs. \#14-17 and \#19), the MR criterion still leads to savings, however the benefit is reduced. In configurations involving primarily strong shock waves and shear layers, both strategies leads to adaptive meshes of similar cell count, particularly Configs. \#11-13 and \#18. In the few Lax-Liu cases, that are dominated by strong and global shock waves (like \#3 and \#4), the SG criterion easily identifies the shock regions and leads to an adaptation with a minimal number of cells.

To better understand the observed differences in criteria performance, we take a closer look into configurations 3, 6, and 10. The used number of cells over all time steps versus the absolute error $L_{1}^{\rho}$ and the adaptation error $L_{1, A M R}^{\rho}$ for both criteria is visualized in Fig. 9. For illustration, the evolution of the number of cells over time for the adaptive MUSCL computation cases listed in Table 1 are displayed in Fig. 10. In these cases the absolute error $L_{1}^{\rho}$ is approximated by using a reference solution evaluated on a highly resolved uniform mesh of $4096 \times 4096$ cells. Compared to Figs. 5 and 6 the behavior is clearly more complex; however, it is eminent that the monotonicity of $L_{1, A M R}^{\rho}$ is preserved in $L_{1}^{\rho}$ and the curves are basically just shifted by the constant value $L_{1, u n i}^{\rho}$. Two cases from each configuration are selected that exhibit a close adaptation error. Characteristic quantities for these cases are listed in Table 1. Unsurprisingly, the time evolution of the number of cells reflect the behavior expressed in the snapshot of the final time in each case described, where the largest growth of number of cells occurs in the 
Table 1: Characteristic adaptation quantities for selected cases with similar $L_{1, A M R}^{\rho}$ error for configurations 3,6 , and 10.

\begin{tabular}{|c|c|c|c|c|c|c|c|c|c|}
\hline \multirow[b]{2}{*}{ LL\# } & & & \multicolumn{4}{|c|}{ MUSCL } & \multicolumn{3}{|c|}{ WENO3 } \\
\hline & & $\begin{array}{c}\epsilon^{\rho} \\
\left(10^{-3}\right)\end{array}$ & $\begin{array}{c}L_{1}^{\rho} \\
\left(10^{-4}\right)\end{array}$ & $\begin{array}{c}L_{1, A M R}^{\rho} \\
\left(10^{-4}\right)\end{array}$ & $\begin{array}{l}\text { Av. } \\
\text { cells } \\
(\%)\end{array}$ & $\begin{array}{c}\text { Final } \\
\text { cells } \\
(\%)\end{array}$ & $\begin{array}{c}L_{1, A M R}^{\rho} \\
\left(10^{-4}\right)\end{array}$ & $\begin{array}{l}\text { Av. } \\
\text { cells } \\
(\%)\end{array}$ & $\begin{array}{l}\text { Final } \\
\text { cells } \\
(\%)\end{array}$ \\
\hline \multirow{2}{*}{3} & $\mathrm{SG}$ & 2.50 & 35.76 & 2.06 & 10.0 & 19.1 & 2.63 & 12.5 & 21.8 \\
\hline & MR & 0.50 & 35.95 & 2.08 & 12.6 & 26.7 & 2.52 & 12.4 & 24.1 \\
\hline \multirow{2}{*}{6} & SG & 5.00 & 95.19 & 7.56 & 31.0 & 81.6 & 5.15 & 31.2 & 76.8 \\
\hline & MR & 0.25 & 93.38 & 7.68 & 22.3 & 45.5 & 6.69 & 24.1 & 47.3 \\
\hline \multirow{2}{*}{10} & $\mathrm{SG}$ & 10.00 & 70.93 & 3.39 & 10.0 & 18.0 & 3.65 & 10.6 & 19.0 \\
\hline & MR & 1.00 & 72.28 & 3.44 & 4.41 & 9.4 & 4.49 & 5.40 & 10.6 \\
\hline
\end{tabular}

shock configuration, while in the rarefaction waves and contacts the MR cases present a much lower growth of the number of cells than the SG cases, even tending to a constant in Config. \#10.

In addition to the average number of cells updated throughout the respective computation relative to the uniform $1024 \times 1024$ case, the relative coverage of cells in the last time step is also listed. For comparison, the results for the same refinement threshold values for the WENO3 method are also given, where we have refrained from approximating $L_{1}^{\rho}$ for this method because of its very high computational expense. The mesh adaptation situation for the three cases at the final time is discussed below. 
Lax-Liu configuration \#3. This test case is dominated primarily by two sharp quasi-one-dimensional shock fronts that propagate almost planar throughout the entire domain, with minor interactions with weak rarefaction waves. The global numerical error varies little as long as the planar shocks are reliably adapted, which can be accomplished with a small number of refinement cells. When the main shocks do interact, a growing rectangular shock and rarefaction pattern is formed; however, its contribution to the global error is comparably small. This somewhat pathological scenario is favorable for the SG criterion, which detects strong shocks very efficiently, as illustrated particularly in the left graphic of Fig. 11. On the other hand, the wavelet basis of the MR criteria is very sensitive to small-scale perturbations of the multi-dimensional shock interaction that are due to the MUSCL scheme. The corresponding WENO3 adaptation pattern (lower, right) is free of these artefacts and hence the average MR efficiency for this configuration is with $\bar{E}_{W E N O 3}=-52.8 \%$ improved compared to $\bar{E}_{M U S C L}=-65.5 \%$ (cf. Fig. 8), albeit still negative. However, note that in absolute values, cf. Fig. 7, this effect is much less pronounced than in relative terms, cf. Fig. 8, and thereby the reduction in computational performance will be less obvious for a user of the AMROC software.

While for this specific test case the benefit of the MR criterion is not apparent in the global error and number of cells, the advantages become obvious when visualizing the local contributions to $L_{1, A M R}(\mathbf{Q})$ according to the summation in Eq. (12). The upper row of Fig. 12 displays the local difference $\left|\mathbf{Q}_{i, j}^{\ell}-\mathbf{Q}_{i, j}^{r}\right|$ for the SG and MR computations of Fig. 11 with the WENO3 method; the lower row visualizes the term $\left|\mathbf{Q}_{i, j}^{\ell}-\mathbf{Q}_{i, j}^{r}\right| \Delta x_{\ell} \Delta y_{\ell}$, 
highlighting the fact that a large error in coarse cells can have a significant influence on an integral norm like $L_{1}$. Comparing Fig. 12 with the refinement patterns in the lower row of Fig. 11, one can discern that large local errors are visible for the SG computation especially along secondary waves that are not refined. On the other hand, the MR criterion captures these waves more gradually and reliably on the second or third mesh adaptation level. As a consequence, the local error (upper row of Fig. 12) shows a much smoother distribution for the MR computation and is free of the perturbations visible in the corresponding plot for the SG calculation, which are a result of fluctuating and insufficient refinement.

Lax-Liu configuration \#6. In this configuration a rotational velocity field with clockwise orientation leads to the creation of swirling shear layers. This effect can clearly be seen in Fig. 13 for both FV schemes. We can observe in the left images of this figure that the SG criterion dramatically overrefines the vortex-like structure plus resulting rarefaction waves to the highest level, while the MR criterion achieves a much better adaptation of density variations of different magnitude. Consequently, the MR criterion achieves a sizable reduction in used cells and a very large reduction in final cells versus SG for all but very small threshold values, cf. Fig. 9 and Table 1. The higher accuracy of the WENO3 method preserves minor vortex and shock structures better, which are accordingly refined by the MR criterion; see right images of Fig. 13. This yields generally smaller $L_{1, A M R}^{\rho}$ errors for the WENO3 scheme for the same threshold choices (cf. Table 1) but also leads to an average MR efficiency that, with $\bar{E}_{W E N O 3}=18.1 \%$, is slightly lower than $\bar{E}_{M U S C L}=25.6 \%$. 
Lax-Liu configuration \# 10. Configuration \#10 is a mixed situation consisting of a shear layer between quadrants $1 / 2$ and $3 / 4$ and Riemann problems with weaker shock and strong rarefactions between $2 / 3$ and $1 / 4$. Very weak secondary shocks or rarefactions are formed in addition due to the interaction of the primary phenomena. As can be seen in the lower row of Fig. 14 the SG criterion struggles considerably to detect these signals of different strength reliably in the density. The MR criterion however captures all of them reliably and on a level of resolution commensurate to the signal gradient. The upper right graphic of Fig. 14 displays the absolute value of wavelet coefficients computed in density alone and all features (highlighted in the upper left graphic by showing the temperature) can be clearly discerned. The larger absolute values of the wavelet coefficients are along the central contact discontinuities; intermediate values detect the regions related to the rarefaction and shock waves and reliably identify the regularity of the numerical solution. In the lower row are again shown the refinement levels generated by the SG and MR criteria with similar $L_{1, A M R}^{\rho}$ error, cf. Table 1 . The SG criterion over-refines the upper right strong rarefaction but misses entire weaker waves. As a result, for this case and the MUSCL scheme the SG criterion will lead to 2.27 times more used cells than the MR criterion to achieve a similar $L_{1, A M R}^{\rho}$ error. With an average MR efficiency of $\bar{E}_{W E N O 3}=28.4 \%$ versus $\bar{E}_{M U S C L}=34.4 \%$ the results for both methods are very similar and the WENO3 results are omitted in Fig. 14 for brevity. However, in Fig. 15 is shown the local integral error contribution $\left|\mathbf{Q}_{i, j}^{\ell}-\mathbf{Q}_{i, j}^{r}\right| \Delta x_{\ell} \Delta y_{\ell}$ for the WENO3 scheme. The graphics highlight that large local errors are again created especially along those secondary waves in the lower left quadrant of 
the domain, which are missed in their entirety by the SG criterion. Hence, the MR criterion achieves a much more homogeneous local error distribution.

\subsection{Three-dimensional ellipsoidally expanding shock wave}

As an example for a gas dynamical problem of larger computational expense, we consider the expansion of an ellipsoidal shock wave in three space dimensions. The Euler equations are solved in the computational domain $\Omega=[-2,2]^{3}$ until the final simulation time $t_{\text {end }}=0.8$. Outflow boundary conditions are applied at all sides of the domain. Initial conditions in density $\rho$ and energy density $\rho E$ are set as

$$
\rho=\left\{\begin{array}{ll}
0.125, & r<r_{c}, \\
1.00, & r \geq r_{c},
\end{array} \quad \rho E= \begin{cases}0.25, & r<r_{c}, \\
2.50, & r \geq r_{c}\end{cases}\right.
$$

with $r_{c}=\frac{3}{5}$, while the velocity vector is initially zero, i.e. $v_{1}=v_{2}=v_{3}=0$ everywhere. The initial ellipsoid is specified by

$$
r=\sqrt{\left(\frac{x_{1_{r}}}{a}\right)^{2}+\left(\frac{x_{2_{r}}}{b}\right)^{2}+\left(\frac{x_{3_{r}}}{c}\right)^{2}}
$$

where

$$
\begin{gathered}
x_{1_{r}}=x_{1} \cos (\theta)-x_{2} \sin (\theta), \\
x_{2_{r}}=\left(x_{1} \sin (\theta)+x_{2} \cos (\theta)\right) \cos (\phi)-x_{3} \sin (\phi), \\
x_{3_{r}}=\left(x_{1} \sin (\theta)+x_{2} \cos (\theta)\right) \sin (\phi)+x_{3} \cos (\phi),
\end{gathered}
$$

with stretching and rotational parameters $a=\frac{1}{3}, b=1, c=3, \theta=\frac{\pi}{3}$, and $\phi=\frac{\pi}{4}$.

The numerical method used is the fully multi-dimensional Wave Propagation Method for Euler equations [23]. In this finite volume method, the 
approximate Riemann solver of Roe in flux difference splitting form is used in face normal direction. Second-order accuracy is achieved by reconstruction and limiting in characteristic variables and blending with the Lax-Wendroff scheme. In addition, a "transverse" Riemann solver of Roe-type is used to approximate cross-derivative fluctuations to second-order accuracy. Here, the complete three-dimensional method is applied. This compute-intensive, but very accurate method approximates three Riemann problems in the normal direction and 36 transverse Riemann problems [40]. Albeit coded in FORTRAN 77, AMROC supports the full wave propagation method as blockbased update scheme, including conservative flux correction at coarse-fine interfaces, cf. [20].

For this test, we use a base mesh of $32^{3}$ cells that can be refined by up to four additional levels with refinement factor 2. This corresponds to a solution on a $512^{3}$ cell mesh in the uniform case. The cluster parameter is always set to $\eta=0.90$ and one buffer cell is added around tagged cells to avoid degradation of results from interpolation. Fixed time steps of $\Delta t=0.04$ are employed on the coarsest level and time step refinement by factor 2 is equally applied throughout all levels, leading to 320 time steps on the finest level as well as in the uniform run. Throughout the computations, the CFL number declines continuously from initially $\sim 0.95$ to $\sim 0.53$. Adaptation is based on evaluating the SG and MR criteria (with hierarchical threshold) in density $\rho$ as well as pressure $p$, where for this problem in non-dimensional quantities the same threshold values are applied to both.

All computations are run in parallel on a single node of a recent Linux compute cluster that provides 20 cores with shared memory. Note however 
Table 2: Characteristic adaptation quantities for two selected cases with similar $L_{1, A M R}^{\rho}$ error for the $3 \mathrm{~d}$ configuration.

\begin{tabular}{ccccccc}
\hline & $\epsilon^{\rho, p}$ & $L_{1, A M R}^{\rho}$ & $\begin{array}{c}\text { Used } \\
\text { cells }\end{array}$ & Final time & $\begin{array}{r}\text { CPU } \\
\text { time }\end{array}$ \\
& $\left(10^{-2}\right)$ & $\left(10^{-2}\right)$ & $\left(10^{9}\right)$ & cells $(\%)$ & \# blocks & $(\mathrm{min})$ \\
\hline $\mathrm{SG}$ & 3.75 & 1.46 & 4.90 & 15.5 & 10963 & 43.5 \\
$\mathrm{MR}$ & 5.00 & 1.42 & 4.02 & 16.7 & 9701 & 39.0 \\
\hline
\end{tabular}

that parallelization in AMROC is through the MPI library. In case of the adaptive computations, dynamic repartitioning to maintain load balance is carried out after each level-0 time step. Computing the uniform solution required $379 \mathrm{~min}$, while the expense of the adaptive computations varies from 4 to $71 \mathrm{~min}$. Two typical computations with similar $L_{1, A M R}^{\rho}$ error for MR and SG criteria are visualized in Figs. 16 and 17. Comparing the cuts in Fig. 17 one notices that the weakening expanding shock and subsequent rarefaction is resolved reliably on the second finest level for MR, while the SG computation alternates between finest and second finest level. On the other hand, the MR criteria pick up complex flow features near the origin that are missed entirely by the SG criteria. Visualizing the wavelet coefficients of the MR computation in Fig. 18 in density and pressure allows us to classify the centermost features as contact discontinuities (as they show no pressure variation) surrounded by shock and/or rarefaction waves. The resulting improved efficiency of the adaptive computation can be seen in Table 2, which shows a benefit of MR in used cells of $18 \%$ and an improvement in actual runtime by $10.4 \%$. This slight difference is due to an increased number of 
refinement blocks in the $\mathrm{MR}$ computations, which raises the overhead in the SAMR method. Measured time spent in the wave propagation scheme is $69 \%$ for the MR and $73.5 \%$ for the SG run. In both computations the evaluation of the criteria themselves is a negligible cost factor.

Finally, Fig. 19 depicts the number of used cells throughout the entire

computation versus the $L_{1, A M R}^{\rho}$ error for SG and MR. Except for very small threshold values, which corresponds to very dense over-refinement, the MR approach always achieves a similar adaptation error with less cells. The average efficiency of MR versus SG, Eq. (18), for Fig. 19 is computed as $\bar{E}=44.1 \%$, while $\Delta \bar{N} / N_{u n i}=1.73 \%$.

\section{Conclusions}

A multiresolution-based mesh adaptation criterion has been implemented in the parallel SAMR framework AMROC. A comprehensive approach has been developed to quantitatively compare the adaptive simulations using the new criterion in terms of numerical error from mesh adaptation and number of cells used with conventional adaptive computations. Here, we have tested the MR criteria for numerous configurations encountered in gas dynamics solving the compressible Euler equations and compared directly to computations using scaled gradient criteria.

It is found that - besides somewhat pathological cases - the MR strategy is far superior to the SG approach. Beside discontinuous shock and contact waves, the MR criteria identify in particular smooth rarefaction waves and their interaction reliably. The MR approach is also extremely robust in detecting even small-scale flow disturbances. In the majority of complex test 
cases considered, the greater mathematical sophistication of the MR criterion leads to smaller approximation errors from dynamic mesh adaptation, while the number of employed cells is reduced, in many realistic cases in $2 \mathrm{~d}$ as well as $3 \mathrm{~d}$ by more than $40 \%$. Yet, the comprehensive investigation in $2 \mathrm{~d}$ uncovered that cases dominated solely by strong shock waves can also be adapted very efficiently by the SG approach. In such technically less relevant scenarios, both approaches easily identify the shock waves. However, due to the wider reaching stencil of the proposed MR criterion a slightly larger refinement region is invariably produced. Nevertheless, this effect will not increase the absolute number of cells considerably and thereby users of adaptive codes will hardly notice it.

A potential next step might be the utilization of the multiresolution prediction as inter-level interpolation in the SAMR method. The present computations used a first-order accurate interpolation method that genuinely avoids over- or undershoots with respect to the coarse level data and therefore remains consistent with the TVD (total variation diminishing) and WENO (weighted essentially non-oscillatory) concepts upon which the shockcapturing methods used in here have been constructed, cf. [36, 23]. The wavelet-based prediction operators do not satisfy such important properties and thereby will have to be combined with a limiting operation to ensure stable numerical results.

In summary, this very promising study motivates future more sophisticated applications of the new class of MR-based mesh refinement indicators, especially in more complex situations. Scenarios, in which simple refinement indicators typically struggle are, for instance, highly perturbed turbulent flow 
fields interacting with weak shock waves or chemically reactive flow, in which gradual combustion is difficult to identify.

\section{Acknowledgments}

The authors thank the FAPESP SPRINT - University of Southampton (Grant: 16/ 50016-9), FAPESP (Grant: 2015/ 25624-2), CNPq (Grant: 306038/ 2015-3), and FINEP (Grant: 0112052700) for support of this research. K. S. thankfully acknowledges financial support from the ANR (Grant: 15-CE40-0019). We also thank Prof. S. Gomes for fruitful discussions that lead to this work.

\section{References}

[1] R. Deiterding, M. Domingues, S. Gomes, K. Schneider, Comparison of adaptive multiresolution and adaptive mesh refinement applied to simulations of the compressible Euler equations., SIAM J. Sci. Comput. 38 (2016) S173-S193.

[2] S. Mallat, A compact multiresolution representation: the wavelet model, in: Proc. IEEE Workshop on Computer Vision (WCV), Miami, FL, 1987, pp. $2-7$.

[3] S. G. Mallat, Multiresolution approximations and wavelet orthonormal bases of $L^{2}(\mathbb{R})$, Transactions of the American Mathematical Society 315 (1989) 69-87.

[4] Y. Meyer, Ondelettes et opérateurs I, Hermann, 1990. 
[5] R. DeVore, Nonlinear approximation, Acta Numerica 7 (1998) 51-150.

[6] S. Müller, Adaptive multiscale schemes for conservation laws, volume 27 of Lectures Notes in Computational Science and Engineering, Springer, Heidelberg, 2003.

[7] K. Schneider, O. V. Vasilyev, Wavelet methods in computational fluid dynamics, Annu. Rev. Fluid. Mech. 42 (2010) 473-503.

[8] M. O. Domingues, S. M. Gomes, O. Roussel, K. Schneider, Adaptive multiresolution methods., ESAIM Proceedings 34 (2011) 1-96.

[9] A. Harten, Multiresolution algorithms for the numerical solution of hyperbolic conservation laws, Comm. Pure Appl. Math. 48 (1995) 13051342 .

[10] A. Cohen, Wavelet methods in numerical analysis, in: P. G. Ciarlet, J. L. Lions (Eds.), Handbook of Numerical Analysis, volume 7, Elsevier, Amsterdam, 2000, pp. 417-711.

[11] G. Chiavassa, R. Donat, Point value multi-scale algorithms for 2D compressible flow, SIAM J. Sci. Comput. 23 (2001) 805-823.

[12] O. Roussel, K. Schneider, A. Tsigulin, H. Bockhorn, A conservative fully adaptive multiresolution algorithm for parabolic PDEs, Journal of Computational Physics 188 (2003) 493 - 523.

[13] R. Abgrall, P. M. Congedo, G. Geraci, G. Iaccarino, An adaptive multiresolution semi-intrusive scheme for uq in compressible fluid problems, 
International Journal for Numerical Methods in Fluids 78 (2015) 595637.

[14] J. D. Calle, P. R. Devloo, S. M. Gomes, Wavelets and adaptive grids for the discontinuous Galerkin method, Numer. Algor. 39 (2005) 143-158.

[15] N. Gerhard, S. Müller, Adaptive multiresolution discontinuous galerkin schemes for conservation laws: multi-dimensional case, Computational and Applied Mathematics 35 (2016) 321-49.

[16] W. Guo, Y. Cheng, An adaptive multiresolution discontinuous galerkin method for time-dependent transport equations in multidimensions, SIAM Journal on Scientific Computing 39 (2017) A2962-92.

[17] N. Gerhard, F. Iacono., G. May., S. Müller, R. Schäfer, A high-order discontinuous galerkin discretization with multiwavelet-based grid adaptation for compressible flows., Journal of Scientific Computing 62 (2015) $25-52$.

[18] D. Rossinelli, B. Hejazialhosseini, W. van Rees, M. Gazzola, M. Bergdorf, P. Koumoutsakos, MRAG-I2D: Multi-resolution adapted grids for remeshed vortex methods on multicore architectures, Journal of Computational Physics. 288 (2015) 1-8.

[19] M. Tanaka, R. Cardoso, H. Bahai, Multi-resolution mps method, Journal of Computational Physics 359 (2018) 106 - 136.

[20] R. Deiterding, Block-structured adaptive mesh refinement - theory, implementation and application, ESAIM Proceedings 34 (2011) 97-150. 
[21] R. Deiterding, M. O. Domingues, Evaluation of multiresolution mesh adaptation criteria in the AMROC framework, in: P. Ivanyi, B. H. V. Topping, G. Varady (Eds.), Proc. 5th Int. Conf. on Parallel, Distributed, Grid and Cloud Computing for Engineering, Civil-Comp Press, 2017, p. Paper 45.

[22] A. Majda, Compressible fluid flow and systems of conservation laws in several space variables, Applied Mathematical Sciences Vol. 53, Springer-Verlag, New York, 1984.

[23] R. J. LeVeque, Finite volume methods for hyperbolic problems, Cambridge University Press, Cambridge, New York, 2002.

[24] M. Berger, J. Oliger, Adaptive mesh refinement for hyperbolic partial differential equations, J. Comput. Phys. 53 (1984) 484-512.

[25] M. Berger, P. Colella, Local adaptive mesh refinement for shock hydrodynamics, J. Comput. Phys. 82 (1988) 64-84.

[26] J. Bell, M. Berger, J. Saltzman, M. Welcome, Three-dimensional adaptive mesh refinement for hyperbolic conservation laws, SIAM J. Sci. Comput. 15 (1994) 127-138.

[27] J. Bell, M. Berger, J. Saltzmann, M. Welcome, Three-dimensional adaptive mesh refinement for hyperbolic conservation laws, SIAM J. Sci. Comput. 15 (1994) 127.

[28] R. Deiterding, Parallel adaptive simulation of multi-dimensional detonation structures, Ph.D. thesis, Brandenburgische Technische Universität Cottbus, 2003. 
[29] R. Deiterding, Construction and application of an AMR algorithm for distributed memory computers, in: T. Plewa, T. Linde, V. G. Weirs (Eds.), Adaptive Mesh Refinement - Theory and Applications, volume 41 of Lecture Notes in Computational Science and Engineering, Springer, 2005, pp. 361-372.

[30] B. L. Bihari, A. Harten, Multiresolution schemes for the numerical solution of 2-D conservation laws I, SIAM J. Sci. Comput. 18 (1996) $315-354$.

[31] S. Donat, R. Lopez-Ureña, High-accuracy approximation of piecewise smooth functions using the truncation and encode approach, Applied Mathematics and Nonlinear Sciences 2 (2017) 367-384.

[32] A. Harten, Multiresolution representation of data: a general framework, SIAM J. Numer. Anal. 33 (1996) 385-394.

[33] R. Abgrall, P. Congedo, G. Geraci, A one-time truncate and encode multiresolution stochastic framework, Journal of Computational Physics 257 (2014) $19-56$.

[34] M. O. Domingues, S. M. Gomes, O. Roussel, K. Schneider, Space-time adaptive multiresolution techniques for compressible euler equations, in: C. A. de Moura, C. S. Kubrusly (Eds.), The Courant-Friedrichs-Lewy (CFL) Condition, Birkhäuser Boston, 2013, pp. 101-117. doi:10.1007/ 978-0-8176-8394-8_7.

[35] A. Cohen, W. Dahmen, R. DeVore, Fully adaptive multiresolution finite volume schemes for conservation laws, Math. Comp. 70 (2001) 27-75. 
[36] E. F. Toro, Riemann solvers and numerical methods for fluid dynamics, Springer, 1999.

[37] P. D. Lax, X. D. Liu, Solution of two-dimensional Riemann problems of gas dynamics by positive schemes, SIAM J. Sci. Comput. 19 (1998) 319-340.

[38] P. L. Roe, Approximate Riemann solvers, parameter vectors, and difference schemes, J. Comput. Phys. 43 (1981) 357-372.

[39] C. Pantano, R. Deiterding, D. J. Hill, D. I. Pullin, A low-numerical dissipation patch-based adaptive mesh refinement method for large-eddy simulation of compressible flows, J. Comput. Phys. 221 (2007) 63-87.

[40] J. Langseth, R. LeVeque, A wave propagation method for three dimensional conservation laws, J. Comput. Phys. 165 (2000) 126-166.

[41] A. Cohen, I. Daubechies, J. C. Feauveau, Biorthogonal bases of compactly supported wavelets, Communications on Pure and Applied Mathematics 45 (1992) 485-560.

[42] S. Mallat, Wavelet tour of signal processing: the sparse way, 3rd ed., Academic Press, New York, 2008.

\section{Appendix A. Cell-average multiresolution and wavelets}

The aim of this appendix is to highlight the equivalence between Harten's discrete MR and continuous biorthogonal wavelets, which is revealing for the readers and not so well-known outside the wavelet community. The cellaverage multiresolution representation introduced by Harten [9, 32] for finite 
volume discretizations of hyperbolic conservation laws is directly related to biorthogonal wavelets. In the following we briefly summarize the concept of multiresolution analysis, its relation to orthogonal and then biorthogonal wavelets. Then we detail the connection between cell-average MR and biorthognal wavelets using the Haar basis. For ease of presentation we choose the one-dimensional scalar-valued case. The extension to higher dimensions using tensor products is given at the end of this section and also how vectorvalued data are treated.

\section{Appendix A.1. Orthogonal wavelets}

Multiresolution analysis introduced by Mallat and Meyer [2, 3, 4] corresponds to a sequence of embedded subspaces, typically denoted by $V^{\ell}$ for $\ell \in \mathbb{N}$, which belong to the Hilbert space of square-integrable functions $L^{2}(\mathbb{R})$. The required inner product reads

$$
\langle f(u), g(u)\rangle=\int_{-\infty}^{\infty} f(u) g(u) d u
$$

and the corresponding norm $\|f\|_{2}=\langle f, f\rangle^{1 / 2}$.

The spaces $V^{\ell}$ have several characteristic properties, in particular they are nested $V^{\ell} \subset V^{\ell+1}$, and a function $q \in L^{2}(\mathbb{R})$ with $q(x) \in V^{\ell}$ satisfies $q(2 x) \in V^{\ell+1}$, which corresponds to contracting the function by a factor two and thus changing the scale. The subspaces $V^{\ell}$ are generated by translated scaling functions $\phi(x)$ which are required to exist. Therewith, we have $V^{\ell}=$

$\operatorname{span}\left\{\phi_{i}^{\ell}\right\}$ where $\phi_{i}^{\ell}(x)=2^{\ell / 2} \phi\left(2^{\ell} x-i\right)$. The nestedness of the subspaces implies that the scaling functions satisfy a refinement equation,

$$
\phi(x)=\sum_{i \in \mathbb{Z}} h_{i} \phi(2 x-i)
$$


In the case that $\phi$ has compact support, the filter coefficients $h_{i}$ (a low pass filter) have only a finite number of non-vanishing coefficients.

In the case of the Haar basis, the scaling function is the characteristic function of the interval $[0,1[$,

$$
\phi(x)=\chi_{[0,1[}(x)= \begin{cases}1 & \text { for } \quad 0 \leq x<1, \\ 0 & \text { elsewhere }\end{cases}
$$

and the filter coefficients are, following the relation

$$
\phi(x)=\phi(2 x)+\phi(2 x-1)
$$

given by $h_{0}=h_{1}=1$ and $h_{i}=0$ elsewhere. This basis function is perfectly adapted to finite volume discretizations as it generates spaces of piecewise constant functions which represent finite volume solutions. Moreover, the Haar scaling function is orthonormal, i.e. $\left\langle\phi_{i}^{\ell}, \phi_{k}^{\ell}\right\rangle=\delta_{i, k}$, and its $L^{2}$-norm yields one, $\left\|\phi_{i}^{\ell}\right\|_{2}=1$. Thus, functions $\phi_{i}^{\ell}$ form an orthonormal basis of $V^{\ell}$.

The nested subspaces can be considered as finite volume approximations at different levels $\ell$ associated to the scales $2^{\ell}$. Wavelets can then be introduced by defining complement spaces $W^{\ell}$ with $V^{\ell+1}=V^{\ell} \oplus W^{\ell}$. Analogously to $V^{\ell}$, which are spanned by the scaling functions $\phi_{i}^{\ell}$, the complement spaces $W^{\ell}$ are spanned by wavelet functions $\psi_{i}^{\ell}$ with $\psi_{i}^{\ell}(x)=2^{\ell / 2} \psi\left(2^{\ell} x-i\right)$. The wavelet also fulfills a refinement equation,

$$
\psi(x)=\sum_{i \in \mathbb{Z}} g_{i} \phi(2 x-i)
$$

with filter coefficients $g_{i}$ (a high pass filter). For the Haar wavelet

$$
\psi(x)=\chi(2 x)-\chi(2 x-1)=\left\{\begin{array}{rll}
1 & \text { for } & 0 \leq x<1 / 2, \\
-1 & \text { for } \quad 1 / 2 \leq x<1 \\
0 & \text { elsewhere }
\end{array}\right.
$$


we have $g_{0}=-g_{1}=1$ and $g_{i}=0$ else, according to

$$
\psi(x)=\phi(2 x)-\phi(2 x-1)
$$

The Haar wavelets are orthonormal and thus satisfy

$$
\left\langle\psi_{i}^{\ell}, \psi_{k}^{\ell^{\prime}}\right\rangle=\delta_{\ell, \ell^{\prime}} \delta_{i, k}
$$

Applying the decomposition $V^{\ell+1}=V^{\ell} \oplus W^{\ell}$ recursively yields a multiresolution analysis of $L^{2}(\mathbb{R})=V^{0} \bigoplus_{\ell=0}^{\infty} W^{\ell}$. A function $q \in L^{2}(\mathbb{R})$ can thus be represented as an orthogonal wavelet series,

$$
q(x)=\sum_{i \in \mathbb{Z}}\left\langle q, \phi_{i}^{0}\right\rangle \phi_{i}^{0}(x)+\sum_{\ell=0}^{\infty} \sum_{i \in \mathbb{Z}}\left\langle q, \psi_{i}^{\ell}\right\rangle \psi_{i}^{\ell}(x),
$$

with the scaling coefficients $\left\langle q, \phi_{i}^{0}\right\rangle$ and the wavelet coefficients $\left\langle q, \psi_{i}^{\ell}\right\rangle$.

\section{Appendix A.2. Biorthogonal wavelets}

Relaxing the orthogonality condition of scaling functions and wavelets implies increased flexibility (in terms of symmetry, number of vanishing moments, filter length, etc.), which is obtained with biorthogonal wavelets [41]. In addition to the scaling function $\phi$ there exists a dual scaling function $\widetilde{\phi}$ which is also refinable with filter coefficients $\widetilde{h}_{i}$. Both functions are biorthogonal to each other, i.e., $\langle\phi(x-i), \widetilde{\phi}(x-j)\rangle=\delta_{i, j} \forall i, j \in \mathbb{Z}$. A dual wavelet $\widetilde{\psi}$ is also required to exist, which fulfills likewise a refinement equation with filter coefficients $\widetilde{g}_{i}$ and which is biorthogonal to the wavelets $\psi$. The filter coefficients are mutually related to each other via $g_{i}=(-1)^{i} \widetilde{h}_{1-i}$ and $\widetilde{g}_{i}=(-1)^{i} h_{1-i}$. 
The biorthogonal wavelet expansion of a function $q \in L^{2}(\mathbb{R})$ thus reads

$$
q(x)=\sum_{i \in \mathbb{Z}}\left\langle q, \widetilde{\phi}_{i}^{0}\right\rangle \phi_{i}^{0}(x)+\sum_{\ell=0}^{\infty} \sum_{i \in \mathbb{Z}}\left\langle q, \widetilde{\psi}_{i}^{\ell}\right\rangle \psi_{i}^{\ell}(x),
$$

and the special case of an orthogonal representation is recovered for $\widetilde{\phi}=\phi$ and $\widetilde{\psi}=\psi$. Note that for a given primary scaling function $\phi$ different dual scaling functions $\widetilde{\phi}$ can be constructed (and vice versa) and thus the choice is not unique.

For the cell-average multiresolution analysis, which is well adapted to finite volumes, the scaling coefficients $\left\langle q, \widetilde{\phi}_{i}^{\ell}\right\rangle$ correspond to the scaled cell average of the cell $\Omega_{i}^{\ell}$. Hence the dual scaling function $\widetilde{\phi}$ is the rescaled Haar scaling function

$$
\widetilde{\phi}_{\ell, i}=\chi_{\Omega_{i}^{\ell}} / \sqrt{\left|\Omega_{i}^{\ell}\right|}
$$

We introduce the cell average ${ }^{1}$ of the cell $\Omega_{i}^{\ell}$ which is defined as $\bar{Q}_{i}^{\ell}=$ $\left\langle q, \tilde{\phi}_{\ell, i}\right\rangle / \sqrt{\left|\Omega_{i}^{\ell}\right|}$. Consequently, we can use the polynomial prediction operator, as the one presented in Eq. 3 and described in [12], to obtain

$$
\widehat{Q}_{2 i}^{\ell+1}=\sum_{n=-s}^{s} \lambda_{n}\left\langle q, \widetilde{\phi}_{i}^{\ell}\right\rangle .
$$

where the details can be computed as

$$
\begin{aligned}
d_{2 i}^{\ell+1} & =\bar{Q}_{2 i}^{\ell+1}-\widehat{Q}_{2 i}^{\ell+1}=\bar{Q}_{2 i}^{\ell+1}-\sum_{n=-s}^{s} \lambda_{n} \bar{Q}_{i+n}^{\ell} \\
& =\left\langle q, \widetilde{\psi}_{i}^{\ell}\right\rangle / \sqrt{\left|\Omega_{i}^{\ell}\right|}
\end{aligned}
$$

\footnotetext{
${ }^{1}$ Note that we denote the cell average by $\bar{Q}_{i}^{\ell}$ instead of $Q_{i}^{\ell}$ in this appendix as it is consistent with our previous work and the notation typically used in the MR community.
} 
The dual wavelet $\widetilde{\psi}_{i}^{\ell}$ is given by a linear combination of dual scaling functions

$$
\widetilde{\psi}_{i}^{\ell}=\widetilde{\phi}_{2 i}^{\ell+1}-\sum_{n=-s}^{s} \lambda_{n} \widetilde{\phi}_{i+n}^{\ell} .
$$

Details for odd indices $d_{2 i+1}^{\ell+1}$ are redundant and equal to $-d_{2 i}^{\ell+1}$. In Harten's MR analysis based on prediction and reconstruction in the discrete framework, wavelet coefficients are determined as linear combinations of prediction errors, whereas in the continuous wavelet context, these coefficients are defined as inner products of the underlying function with the wavelets. The connection between these ideas is presented in Eq. A.9.

The filter coefficients of the biorthogonal multiresolution can thus be directly computed and we find $\widetilde{h}_{0}=\widetilde{h}_{1}=1$ and $\widetilde{h}_{i}=0$ elsewhere, corresponding to the Haar scaling function. Using the relation with the filter coefficients of $g$ we find $g_{0}=-g_{1}=1$ and $g_{i}=0$ elsewhere. Choosing the prediction operator with $r=2 s+1=3$ and the values $\lambda_{-1}=-\lambda_{1}=-1 / 8, \lambda_{0}=0$ we obtain the filter coefficients $h_{0}=h_{1}=1, h_{-1}=h_{2}=1 / 8, h_{-2}=h_{3}=-1 / 8$ and $h_{i}=0$ elsewhere. Correspondingly, we find for $\widetilde{g}_{0}=1, \widetilde{g}_{1}=-1, \widetilde{g}_{2}=$ $\widetilde{g}_{3}=1 / 8, \widetilde{g}_{-1}=\widetilde{g}_{-2}=-1 / 8$ and $\widetilde{g}_{i}=0$ elsewhere. Plots of the corresponding biorthogonal scaling and wavelet functions are presented in Fig. A.20.

Vanishing moments or polynomial cancellation proprieties of wavelets. A wavelet function has $p$ vanishing moments if and only if its related scaling function can generate polynomials up to degree $p-1$. Therefore, the wavelet coefficients are zero for polynomials of degree at most $p-1$, so that, the scaling functions alone can be used to represent the function. More vanishing moments of the wavelet function imply that its related scaling function can represent more complex functions and that the wavelet representation is 
sparser, as many wavelet coefficients vanish. In Fourier space this property is equivalent to say that the first $p$ derivatives of the Fourier transform of the wavelet function of the wavelet function vanish at frequency zero. The cancellation property of wavelets is an essential ingredient to obtain a sparse representation of functions which are locally smooth. This motivates the thresholding procedure and justifies that the MR criteria can be considered as a shock detector.

Boundary conditions. Harten's MR analysis for cell averages is adapted to bounded domains, as the analyzing scaling function does correspond to the indicator function and is thus adapted to the interval. Hence no special care is required to take into account boundary conditions on Cartesian domains.

Extensions for high space dimension. Extensions are obtained by tensor product, cf. Appendix A of [12]. In two dimensions the spaces $\mathbf{V}^{\ell}$ are constructed via $\mathbf{V}^{\ell}=V^{\ell} \otimes V^{\ell}$. Using $V^{\ell}=V^{\ell-1} \oplus W^{\ell-1}$, we thus obtain $\mathbf{V}^{\ell}=\left(V^{\ell-1} \oplus W^{\ell-1}\right) \otimes\left(V^{\ell-1} \oplus W^{\ell-1}\right)=V^{\ell-1} \otimes V^{\ell-1} \oplus W^{\ell-1} \otimes V^{\ell-1} \oplus$ $V^{\ell-1} \otimes W^{\ell-1} \oplus W^{\ell-1} \otimes W^{\ell-1}=\mathbf{V}^{\ell-1} \oplus \mathbf{W}^{\ell-1}$, where $\mathbf{W}^{\ell-1}$ corresponds to three wavelet spaces in the horizontal, vertical and diagonal directions. The procedure in three dimensions is analogous and yields seven directions. The construction of the corresponding biorthogonal wavelet basis in $2 \mathrm{~d}$ and $3 \mathrm{~d}$ is likewise obtained by tensor product and the resulting wavelets have consequently three and seven directions in $2 \mathrm{~d}$ and $3 \mathrm{~d}$, respectively. For details we refer to [42].

Vector-valued functions. The biorthogonal wavelet expansion is applied to each component of the vector and thus a vector-valued wavelet series is ob- 
tained as the coefficients become vector-valued, but not the basis functions.

\section{Appendix B. Lax-Liu configurations}

The Lax-Liu benchmarks, in [37], are classical 2d Riemann problems of gas dynamics. The computational domain is a square $\Omega=[0,1]^{2}$ and the initial condition is constant within the four quadrants denoted by $1,2,3$, and 4, as illustrated in Fig. B.21. For the sake of comparison with our results and other, we also use the same final times described in [37], cf. Table B.3. In Table B.4 we display in a more systematic form the initial conditions used on these sub-domains. We also indicate for each configuration the formation of Rarefaction waves (R), Contact Discontinuities (J), and Shocks (S) that arise in the fluid interfaces. The arrow direction $(\rightarrow, \leftarrow)$ and the inclinations (with the symbols \pm ) complement the flow structure developed.

Table B.3: Final time used in the Lax-Liu simulations.

\begin{tabular}{lcccccccccc}
\hline & \multicolumn{11}{c}{ Lax Liu configurations } \\
Number & 1 & 2 & 3 & 4 & 5 & 6 & 7 & 8 & 9 & 10 \\
$t_{\text {end }}$ & 0.20 & 0.20 & 0.30 & 0.25 & 0.30 & 0.30 & 0.25 & 0.25 & 0.30 & 0.15 \\
\hline Number & 11 & 12 & 13 & 14 & 15 & 16 & 17 & 18 & 19 & \\
$t_{\text {end }}$ & 0.30 & 0.25 & 0.30 & 0.10 & 0.02 & 0.20 & 0.30 & 0.20 & 0.30 & \\
\hline
\end{tabular}



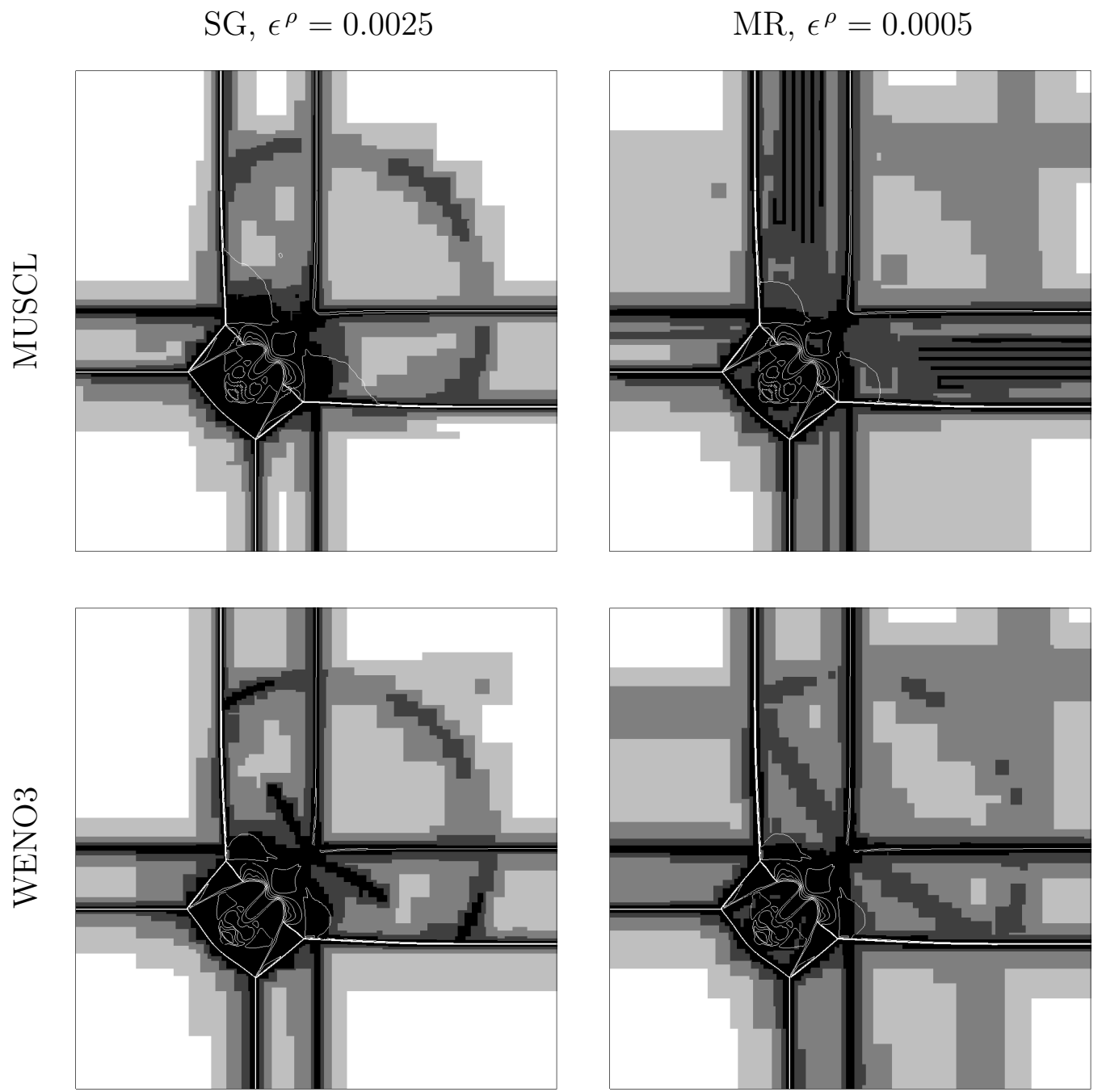

Figure 11: Lax-Liu Config. \# 3: density contours for the SG and MR computations of Table 1 superimposed on refinement levels (in gray scales) at final time $t_{\text {end }}=0.3$. 

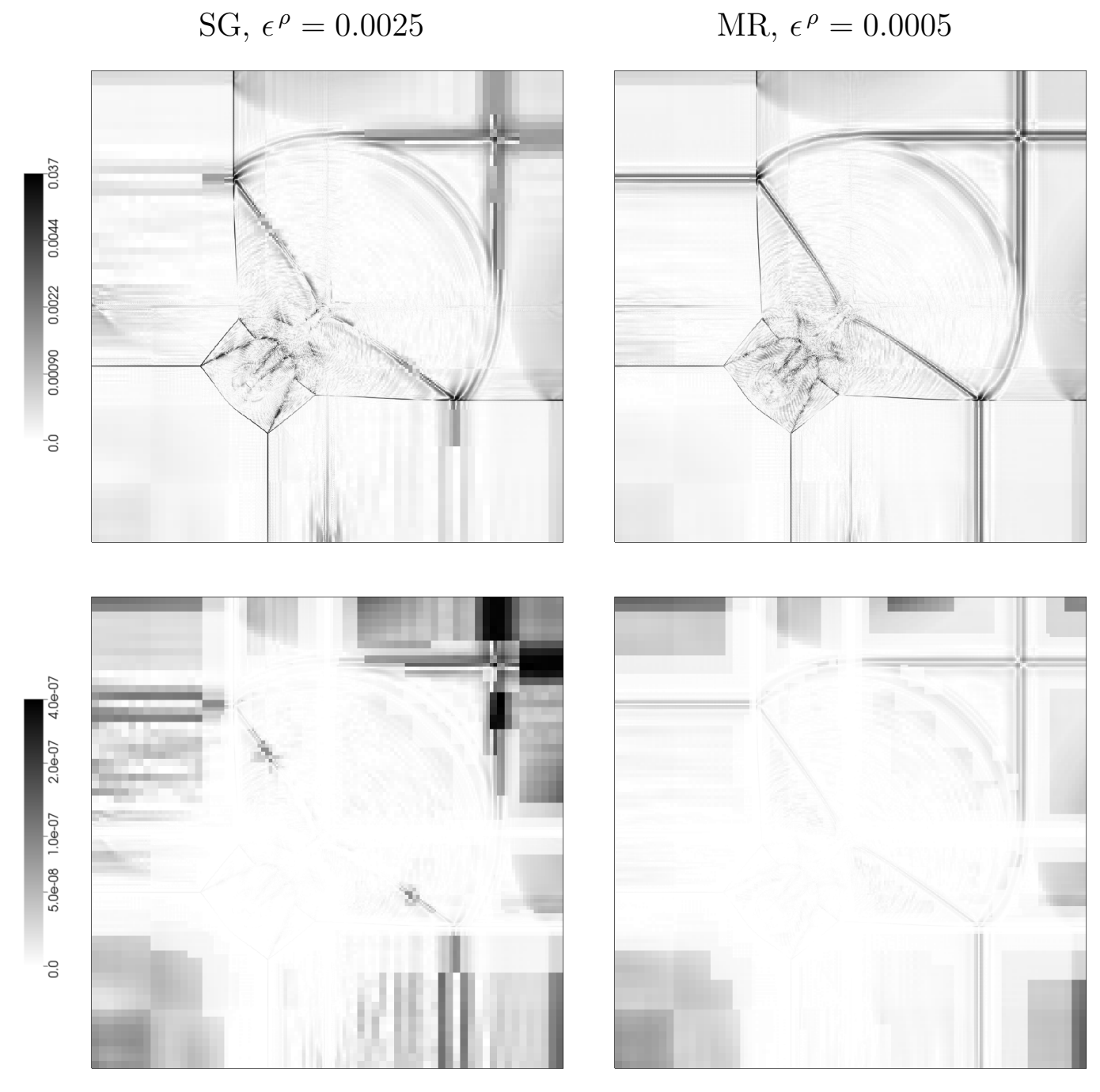

Figure 12: Lax-Liu Config. \# 3: Local error $\left|\mathbf{Q}_{i, j}^{\ell}-\mathbf{Q}_{i, j}^{r}\right|$ (upper row) and

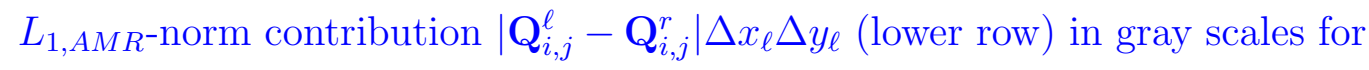
the SG and MR computations of Fig. 11 with WENO3 at final time $t_{\text {end }}=0.3$. 

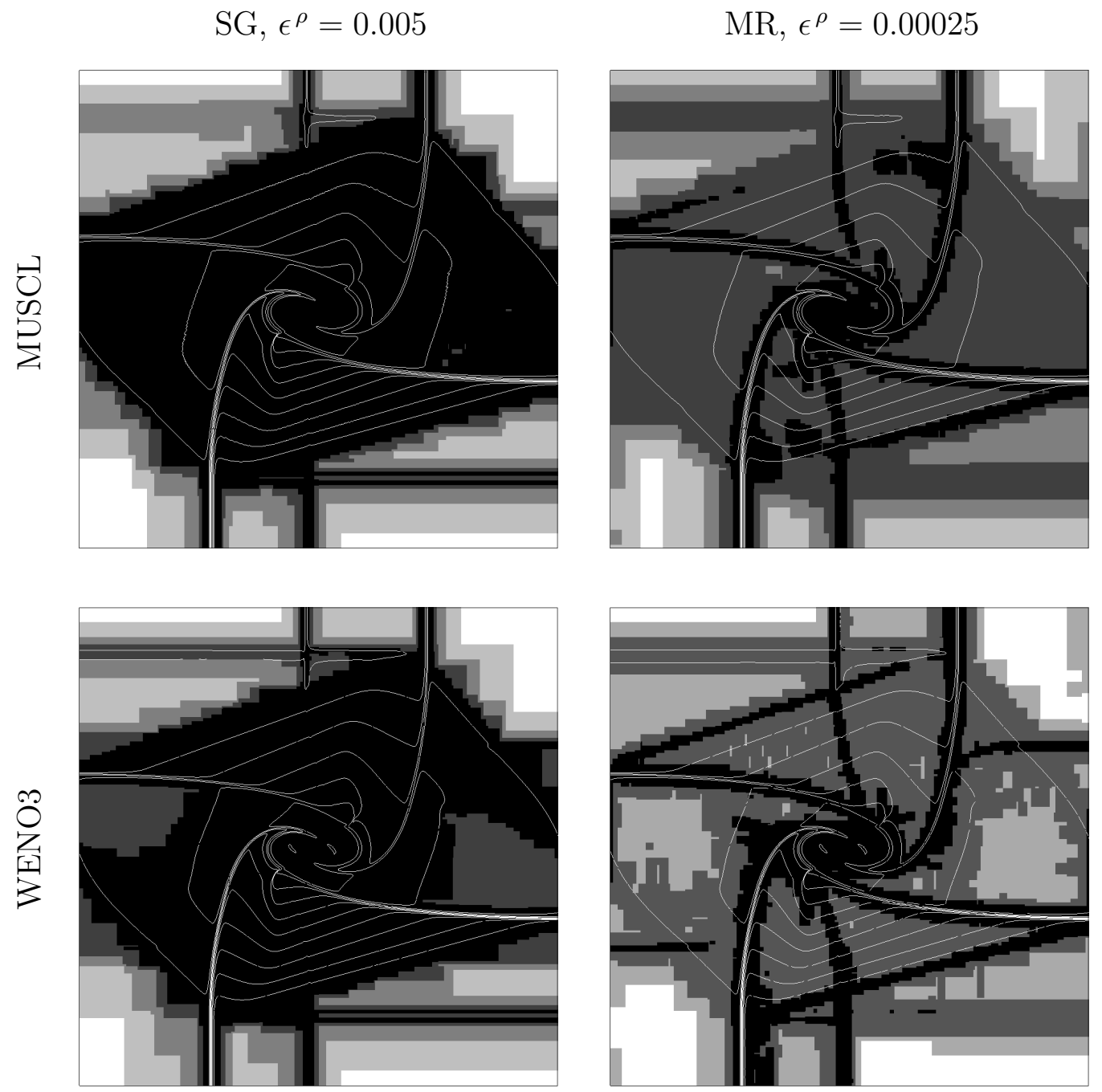

Figure 13: Lax-Liu Config. \# 6: density contours for the SG and MR computations of Table 1 superimposed on refinement levels (in gray scales) at final time $t_{\text {end }}=0.3$. 
Temperature

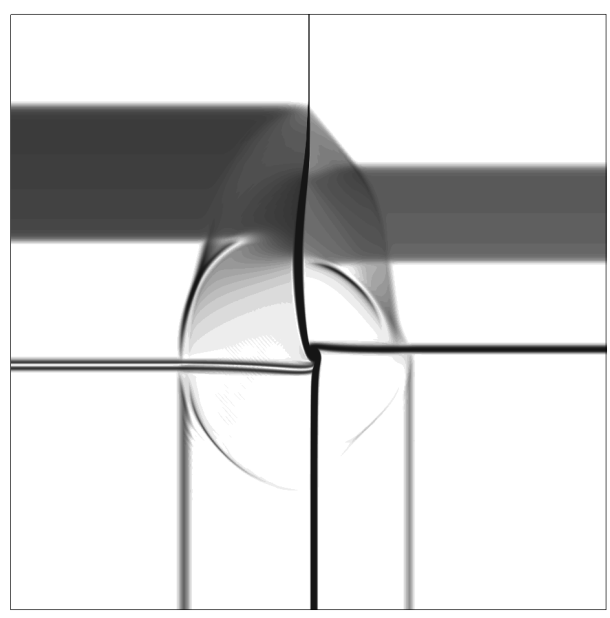

$\mathrm{SG}, \epsilon^{\rho}=0.010$

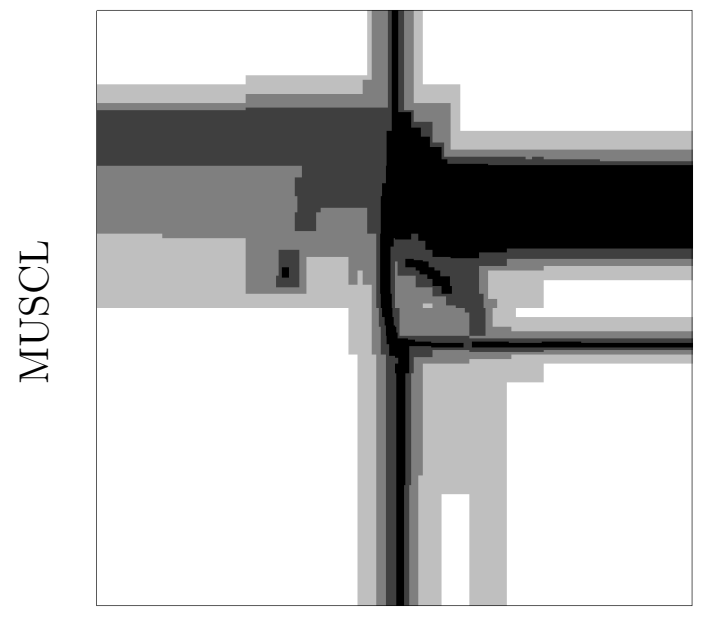

abs. wavelet coefficients

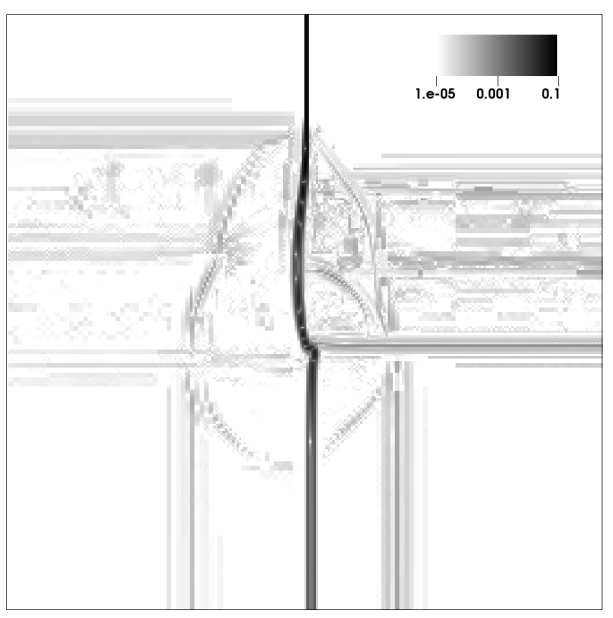

$\mathrm{MR}, \epsilon^{\rho}=0.001$

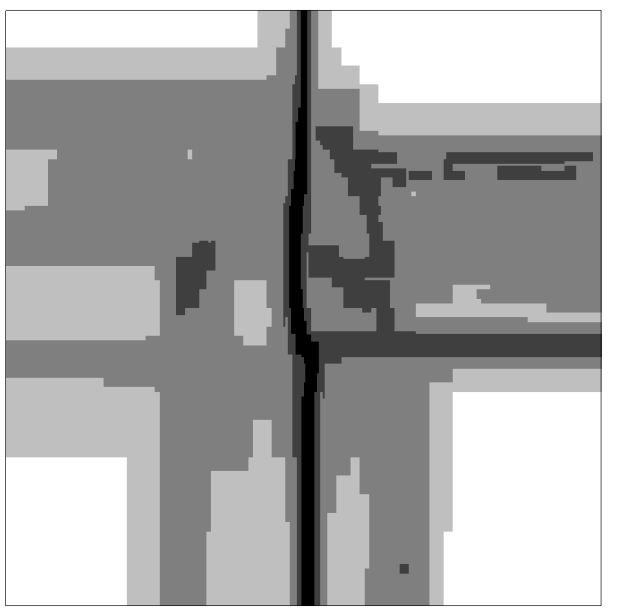

Figure 14: Lax-Liu Config. \#10: Schlieren plot of temperature (top, left), gray scale plots of wavelet coefficients (top, right) and refinement levels (bottom) at final time $t_{\text {end }}=0.15$. 

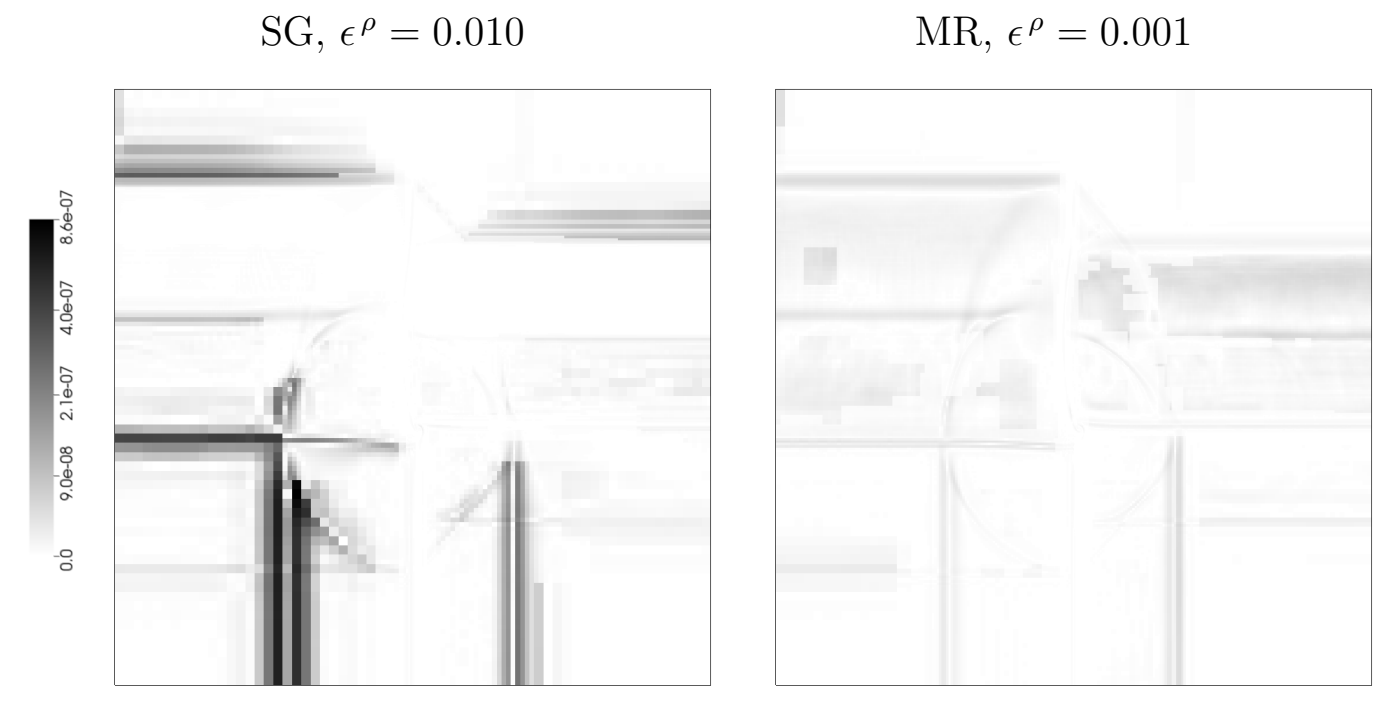

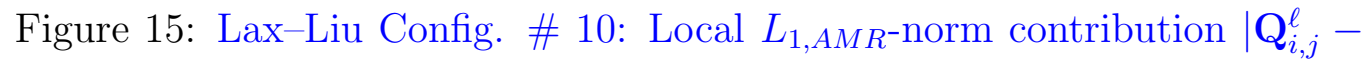
$\mathrm{Q}_{i, j}^{r} \mid \Delta x_{\ell} \Delta y_{\ell}$ in gray scales for the SG and MR computations of Fig. 14 with WENO3 at final time $t_{\text {end }}=0.15$.
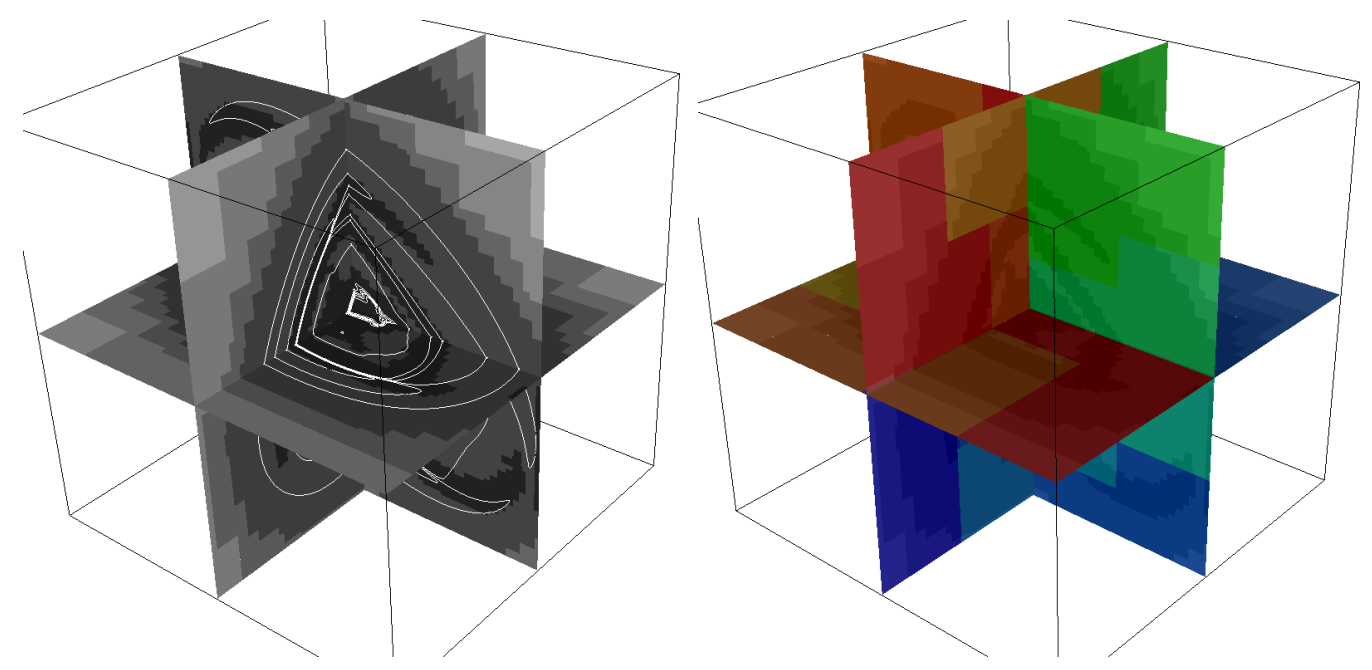

Figure 16: Contours of density shown on levels of mesh refinement in $3 \mathrm{~d}$ (left) for MR with $\epsilon^{\rho, p}=0.05$ at $t_{\text {end }}=0.8$. The right graphic indicates by color the distribution to 20 processors at this time. 


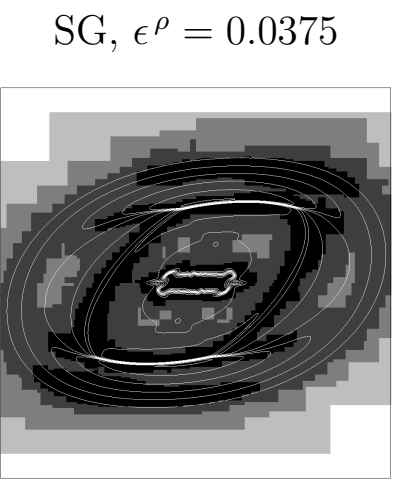

$\mathrm{MR}, \epsilon^{\rho, p}=0.05$
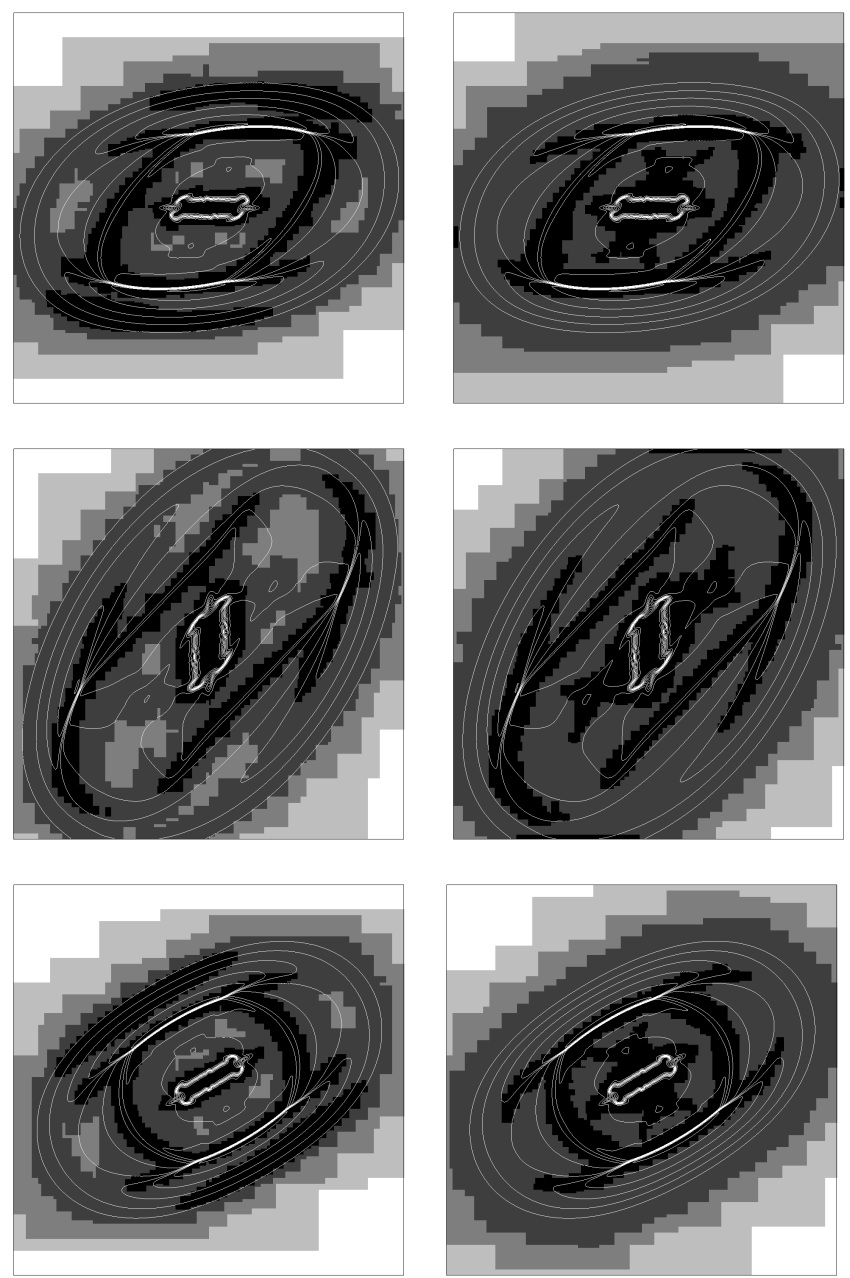

Figure 17: Adaptation for the $3 \mathrm{~d}$ shock-wave case at the time $t_{\text {end }}=0.8$. Isolines of two-dimensional cuts of density for the SG and MR computation superimposed on domain of refinement (in gray scales). From top to bottom: $y-z$-plane at $x=0, x-z$-plane at $y=0, x-y$-plane $z=0$. 

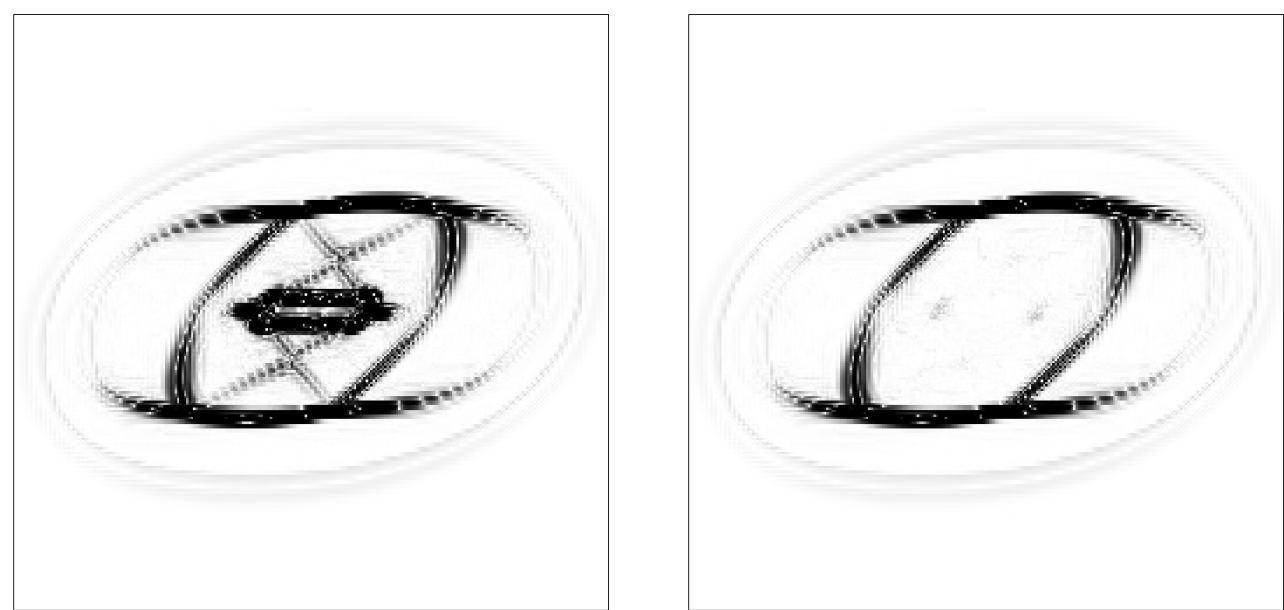

Figure 18: Visualization of wavelet coefficients in $x$-plane at $t=0.72$ in density (left) and pressure (right).

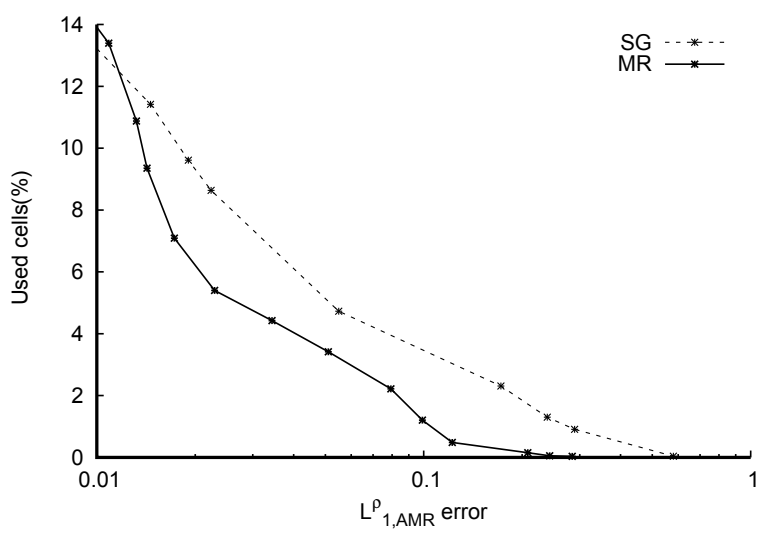

Figure 19: Used cells in the adaptive $3 \mathrm{~d}$ computations versus $L_{1, A M R}^{\rho}$ error at the time $t_{\mathrm{e}}=0.8$. 

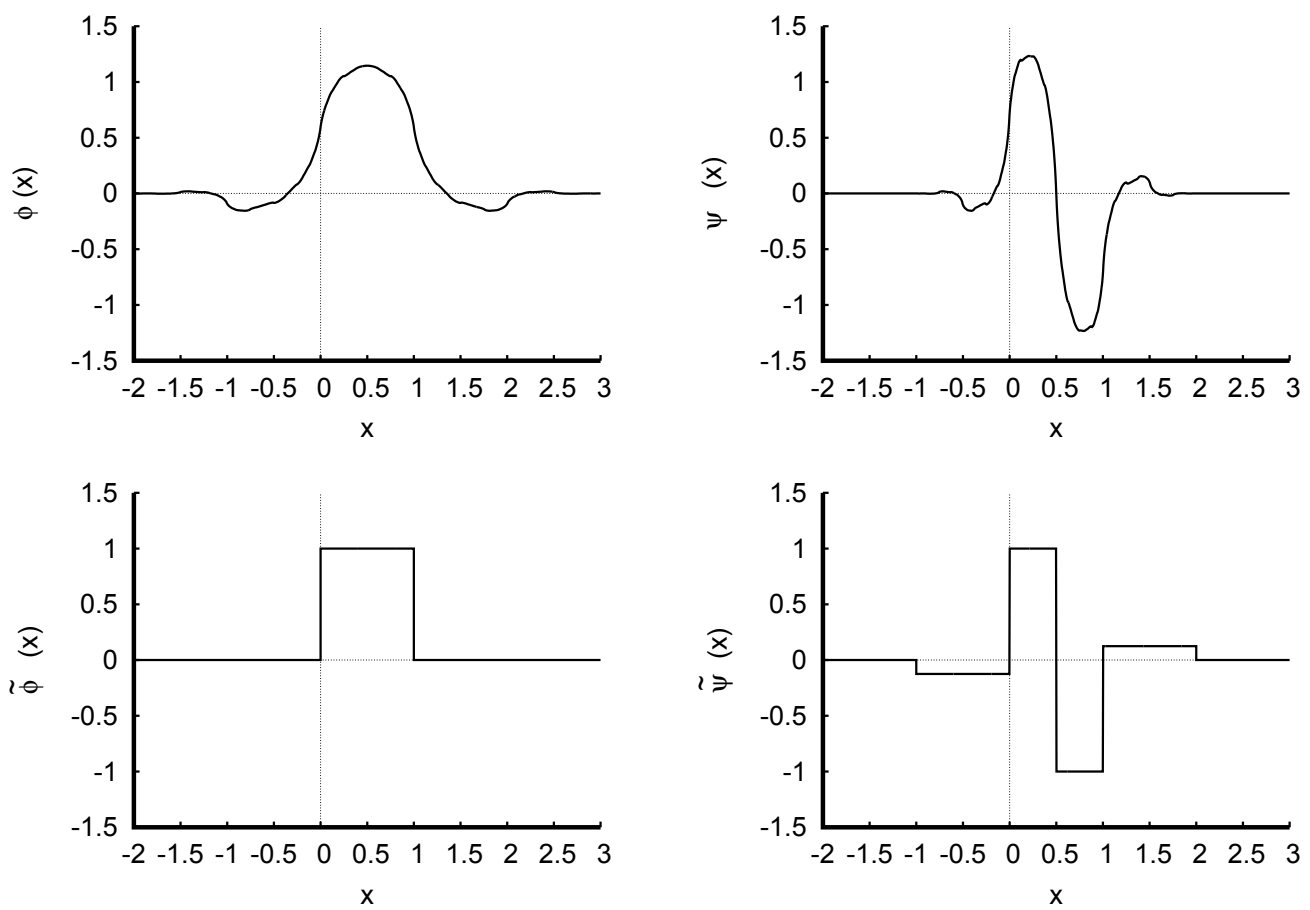

Figure A.20: Biorthogonal scaling functions $\phi$ and $\widetilde{\phi}$ (left, top and bottom), and wavelet functions $\psi$ and $\widetilde{\psi}$ (right, top and bottom) for $r=3$.

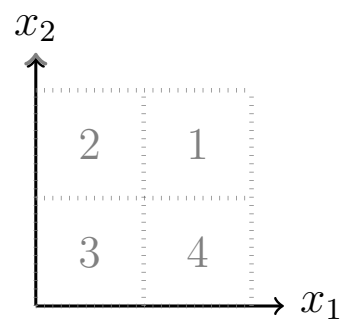

Figure B.21: Initial domain partition for the Lax-Liu configurations. 
Table B.4: Initial conditions for Lax-Liu configurations \#1 - 19.

\begin{tabular}{|c|c|c|c|c|c|c|c|c|c|c|}
\hline \multirow[t]{2}{*}{$Q$} & \multirow[t]{2}{*}{ Configuration } & \multicolumn{4}{|c|}{ Domain position } & \multirow[t]{2}{*}{ Configuration } & \multicolumn{4}{|c|}{ Domain position } \\
\hline & & 1 & 2 & 3 & 4 & & 1 & 2 & 3 & 4 \\
\hline$\rho$ & Number 1 & 1.0000 & 0.5197 & 0.1072 & 0.2579 & Number 11 & 1.0000 & 0.5313 & 0.8000 & 0.5313 \\
\hline$p$ & $\vec{R}_{21}$ & 1.0000 & 0.4000 & 0.0439 & 0.1500 & $\stackrel{\leftarrow}{S}_{21}$ & 1.0000 & 0.4000 & 0.4000 & 0.4000 \\
\hline$v_{1}$ & $\vec{R}_{32} \quad \vec{R}_{41}$ & 0.0000 & -0.7259 & -0.7259 & 0.0000 & $J_{32}^{+} \quad \overleftarrow{S}_{41}$ & 0.1000 & 0.8276 & 0.1000 & 0.1000 \\
\hline$v_{2}$ & $\vec{R}_{34}$ & 0.0000 & 0.0000 & -1.4045 & -1.4045 & $J_{34}^{+}$ & 0.0000 & 0.0000 & 0.0000 & 0.7276 \\
\hline$\rho$ & Number 2 & 1.0000 & 0.5197 & 1.0000 & 0.5197 & Number 12 & 0.5313 & 1.0000 & 0.8000 & 1.0000 \\
\hline$p$ & $\vec{R}_{21}$ & 1.0000 & 0.4000 & 1.0000 & 0.4000 & $\vec{S}_{21}$ & 1.0000 & 0.4000 & 0.4000 & 0.4000 \\
\hline$v_{1}$ & $\stackrel{\leftarrow}{R}_{32} \quad \vec{R}_{41}$ & 0.0000 & -0.7259 & -0.7259 & 0.0000 & $J_{32}^{+} \quad \vec{S}_{41}$ & 0.0000 & 0.7276 & 0.0000 & 0.0000 \\
\hline$v_{2}$ & $\stackrel{\leftarrow}{R}_{34}$ & 0.0000 & 0.0000 & -0.7259 & -0.7259 & $J_{34}^{+}$ & 0.0000 & 0.0000 & 0.0000 & 0.7276 \\
\hline$\rho$ & Number 3 & 1.5000 & 0.5323 & 0.1380 & 0.5323 & Number 13 & 1.0000 & 2.0000 & 1.0625 & 0.5313 \\
\hline$p$ & $\stackrel{\leftarrow}{S}_{21}$ & 1.5000 & 0.3000 & 0.0290 & 0.3000 & $J_{21}^{-}$ & 1.0000 & 1.0000 & 0.4000 & 0.4000 \\
\hline$v_{1}$ & $\overleftarrow{S}_{32} \quad \overleftarrow{S}_{41}$ & 0.0000 & 1.2060 & 1.2060 & 0.0000 & $\stackrel{\leftarrow}{S}_{32} \quad \stackrel{\leftarrow}{S}_{41}$ & 0.0000 & 0.0000 & 0.0000 & 0.0000 \\
\hline$v_{2}$ & $\overleftarrow{S}_{34}$ & 0.0000 & 0.0000 & 1.2060 & 1.2060 & $J_{34}^{-}$ & -0.3000 & 0.3000 & 0.8145 & 0.4276 \\
\hline$\rho$ & Number 4 & 1.1000 & 0.5065 & 1.1000 & 0.5065 & Number 14 & 2.0000 & 1.0000 & 0.4736 & 0.9474 \\
\hline$p$ & $\overleftarrow{S}_{21}$ & 1.1000 & 0.3500 & 1.1000 & 0.3500 & $J_{21}^{+}$ & 8.0000 & 8.0000 & 2.6667 & 2.6667 \\
\hline$v_{1}$ & $\vec{S}_{32} \quad \overleftarrow{S}_{41}$ & 0.0000 & 0.8939 & 0.8939 & 0.0000 & $\overleftarrow{S}_{32} \overleftarrow{S}_{41}$ & -0.5606 & -1.2172 & 1.2172 & 1.1606 \\
\hline$v_{2}$ & $\vec{S}_{34}$ & 0.0000 & 0.0000 & 0.8939 & 0.8939 & $J_{34}^{-}$ & -0.3000 & 0.3000 & 0.8145 & 0.4276 \\
\hline$\rho$ & Number 5 & 1.0000 & 2.0000 & 1.0000 & 3.0000 & Number 15 & 1.0000 & 0.5197 & 0.8000 & 0.5313 \\
\hline$p$ & $J_{21}^{-}$ & 1.0000 & 1.0000 & 1.0000 & 1.0000 & $\vec{R}_{21}$ & 1.0000 & 0.4000 & 0.4000 & 0.4000 \\
\hline$v_{1}$ & $J_{32}^{-} \quad J_{41}^{-}$ & -0.7500 & -0.7500 & 0.7500 & 0.7500 & $J_{32}^{-} \quad \overleftarrow{S}_{41}$ & 0.1000 & -0.6259 & 0.1000 & 0.1000 \\
\hline$v_{2}$ & $J_{34}^{-}$ & -0.5000 & 0.5000 & 0.5000 & -0.5000 & $J_{34}^{+}$ & -0.3000 & -0.3000 & -0.3000 & 0.4276 \\
\hline$\rho$ & Number 6 & 1.0000 & 2.0000 & 1.0000 & 3.0000 & Number 16 & 0.5313 & 1.0222 & 0.8000 & 1.000 \\
\hline$P$ & $J_{21}^{-}$ & 1.0000 & 1.0000 & 1.0000 & 1.0000 & $\stackrel{\leftarrow}{R}_{21}$ & 0.4000 & 1.0000 & 1.0000 & 1.0000 \\
\hline$v_{1}$ & $\begin{array}{ll}J_{32}^{+} & J_{41}^{+}\end{array}$ & 0.7500 & 0.7500 & -0.7500 & -0.7500 & $J_{32}^{-} \quad \vec{S}_{41}$ & 0.1000 & -0.6179 & 0.1000 & 0.1000 \\
\hline$v_{2}$ & $J_{34}^{-}$ & -0.5000 & 0.5000 & 0.5000 & -0.5000 & $J_{34}^{+}$ & 0.1000 & 0.1000 & 0.1000 & 0.8276 \\
\hline$\rho$ & Number 7 & 1.0000 & 0.5197 & 0.8000 & 0.5197 & Number 17 & 1.000 & 2.0000 & 1.0625 & 0.5197 \\
\hline$p$ & $\vec{R}_{21}$ & 1.0000 & 0.4000 & 0.4000 & 0.4000 & $J_{21}^{-}$ & 1.0000 & 1.0000 & 0.4000 & 0.4000 \\
\hline$v_{1}$ & $J_{32}^{-} \quad \vec{R}_{41}$ & 0.1000 & -0.6259 & 0.1000 & 0.1000 & $\overleftarrow{S}_{32} \quad \vec{S}_{41}$ & 0.0000 & 0.0000 & 0.0000 & 0.0000 \\
\hline$v_{2}$ & $J_{34}^{-}$ & 0.1000 & 0.1000 & 0.1000 & -0.6259 & $J_{34}^{-}$ & -0.4000 & -0.3000 & 0.2145 & -1.1259 \\
\hline$\rho$ & Number 8 & 0.5197 & 1.0000 & 0.8000 & 1.0000 & Number 18 & 1.000 & 2.0000 & 1.0625 & 0.5197 \\
\hline$p$ & $\vec{R}_{21}$ & 0.4000 & 1.0000 & 1.0000 & 1.0000 & $J_{21}^{+}$ & 1.0000 & 1.0000 & 0.4000 & 0.4000 \\
\hline$v_{1}$ & $J_{32}^{-} \quad \stackrel{\leftarrow}{R}_{41}$ & 0.1000 & -0.6259 & 0.1000 & 0.1000 & 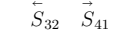 & 0.0000 & 0.0000 & 0.0000 & 0.0000 \\
\hline$v_{2}$ & $J_{34}^{-}$ & 0.1000 & 0.1000 & 0.1000 & -0.6259 & $J_{34}^{+}$ & 1.0000 & -0.3000 & 0.2145 & 0.2741 \\
\hline$\rho$ & Number 9 & 1.0000 & 2.0000 & 1.0390 & 0.5197 & Number 19 & 1.000 & 2.0000 & 1.0625 & 0.5197 \\
\hline$p$ & $J_{21}^{+}$ & 1.0000 & 1.0000 & 0.4000 & 0.4000 & $J_{21}^{+}$ & 1.0000 & 1.0000 & 0.4000 & 0.4000 \\
\hline$v_{1}$ & $\vec{R}_{32} \quad \vec{R}_{41}$ & 0.0000 & 0.000 & 0.0000 & 0.0000 & $\overleftarrow{S}_{32} \quad \vec{S}_{41}$ & 0.0000 & 0.0000 & 0.0000 & 0.0000 \\
\hline$v_{2}$ & $J_{34}^{+}$ & 0.3000 & -0.3000 & -0.8133 & -0.4259 & $J_{34}^{-}$ & 0.3000 & -0.3000 & 0.0000 & 0.0000 \\
\hline$\rho$ & Number 10 & 1.0000 & 0.5000 & 0.2281 & 0.4562 & & & & & \\
\hline$p$ & $J_{21}^{-}$ & 1.0000 & 1.0000 & 0.3333 & 0.3333 & & & & & \\
\hline$v_{1}$ & $\vec{R}_{32} \quad \vec{R}_{41}$ & 0.0000 & 0.0000 & 0.0000 & 0.1000 & & & & & \\
\hline$v_{2}$ & $J_{34}^{+}$ & 0.4297 & 0.6076 & -0.6076 & -0.4297 & & & & & \\
\hline
\end{tabular}

